\title{
A Physical Map of the Short Arm of Wheat Chromosome
} $1 \mathrm{~A}$

\author{
James Breen ${ }^{19}$, Thomas Wicker ${ }^{19}$, Margarita Shatalina', Zeev Frenkel ${ }^{2}$, Isabelle Bertin ${ }^{3}$, \\ Romain Philippe ${ }^{3}$, Wolfgang Spielmeyer ${ }^{4}$, Hana Šimková ${ }^{5}$, Jan Šafáŕ5 ${ }^{5}$, Federica Cattonaro ${ }^{6}$, \\ Simone Scalabrin ${ }^{6}$, Federica Magni ${ }^{6}$, Sonia Vautrin ${ }^{7}$, Hélène Bergès ${ }^{7}$, International Wheat Genome \\ Sequencing Consortium ", Etienne Paux ${ }^{3}$, Tzion Fahima ${ }^{2}$, Jaroslav Doležel ${ }^{5}$, Abraham Korol ${ }^{2}$, \\ Catherine Feuillet ${ }^{3}$, Beat Keller ${ }^{1 *}$
}

1 Institute of Plant Biology, University of Zurich, Zurich, Switzerland, 2 Institute of Evolution, University of Haifa, Haifa, Israel, 3 INRA UMR 1095, Genetique Diversite et Ecophysiologie des Cereales, Clermont-Ferrand, France, 4 CSIRO Plant Industry, Canberra, Australia, 5 Centre of the Region Hana for Biotechnological and Agricultural Research, Institute of Experimental Botany, Olomouc, Czech Republic, 6 Istituto di Genomica Applicata, Udine, Italy, 7 CNRGV - INRA, Castanet Tolosan, France

\begin{abstract}
Bread wheat (Triticum aestivum) has a large and highly repetitive genome which poses major technical challenges for its study. To aid map-based cloning and future genome sequencing projects, we constructed a BAC-based physical map of the short arm of wheat chromosome 1A (1AS). From the assembly of 25,918 high information content (HICF) fingerprints from a 1AS-specific BAC library, 715 physical contigs were produced that cover almost $99 \%$ of the estimated size of the chromosome arm. The 3,414 BAC clones constituting the minimum tiling path were end-sequenced. Using a gene microarray containing $\sim 40 \mathrm{~K} \mathrm{NCBI}$ UniGene EST clusters, PCR marker screening and BAC end sequences, we arranged 160 physical contigs ( $97 \mathrm{Mb}$ or $35.3 \%$ of the chromosome arm) in a virtual order based on synteny with Brachypodium, rice and sorghum. BAC end sequences and information from microarray hybridisation was used to anchor $3.8 \mathrm{Mbp}$ of Illumina sequences from flow-sorted chromosome 1AS to BAC contigs. Comparison of genetic and synteny-based physical maps indicated that $\sim 50 \%$ of all genetic recombination is confined to $14 \%$ of the physical length of the chromosome arm in the distal region. The 1AS physical map provides a framework for future genetic mapping projects as well as the basis for complete sequencing of chromosome arm $1 \mathrm{AS}$.
\end{abstract}

Citation: Breen J, Wicker T, Shatalina M, Frenkel Z, Bertin I, et al. (2013) A Physical Map of the Short Arm of Wheat Chromosome 1A. PLoS ONE 8(11): e80272. doi:10.1371/journal.pone.0080272

Editor: Turgay Unver, Cankiri Karatekin University, Turkey

Received May 8, 2013; Accepted October 11, 2013; Published November 21, 2013

Copyright: (c) 2013 Breen et al. This is an open-access article distributed under the terms of the Creative Commons Attribution License, which permits unrestricted use, distribution, and reproduction in any medium, provided the original author and source are credited.

Funding: The research leading to these results received funding from the University of Zurich and the European Community's Seventh Framework Programme (FP7/2007-2013) under the grant agreement FP7-212019 (Triticeae Genome). The funders had no role in study design, data collection and analysis, decision to publish, or preparation of the manuscript.

Competing Interests: The authors have declared that no competing interests exist.

*E-mail: bkeller@botinst.uzh.ch

9 These authors contributed equally to this work.

- Membership of the International Wheat Consortium (IWGSC) is provided at wheatgenome.org/Organization/General-members-list

\section{Introduction}

The Poaceae family of grass species includes some of the most economically important agricultural crop species in the world. Plants such as rice (Oryza sativa), sorghum (Sorghum bicolor), maize (Zea mays), wheat (Triticum aestivum) and barley (Hordeum vulgare) provide a large share of the world's food. In order to increase crop productivity, genome sequencing projects have been initiated to provide genetic tools for plant improvement [1]. Grass species such as rice [2], sorghum [3], Brachypodium distachyon [4] have had their genomes completely sequenced. For barley, a partial genome sequence became recently available which covers gene space but is incomplete in repetitive intergenic regions [5].

Model genomes such as rice [2], sorghum [3] and Brachypodium [5] have been used to infer gene order in wheat and barley: positional information of genes from syntenic regions in these three model genomes was compiled for the production of "chromosome zippers" [6]. These were used to deduce a virtual gene order of the barley genome [7] and wheat group 1 chromosomes [8]. However, these studies as well as sequencing of large contigs of chromosome 3B [9] also showed that both wheat and barley contain approximately $50 \%$ of non-colinear genes which cannot be ordered based on synteny to model genomes. Thus, the production of physical maps and ultimately a complete genome sequence are essential for map-based cloning and to determine the correct order of genes. In wheat however, genome sequencing efforts are hampered by a large $17,000 \mathrm{Mb}$ genome size (over 43 times larger than the rice genome), $>80 \%$ repetitive sequence $[10,11]$ and hexaploid (AABBDD) genome structure.

One of the most important resources for physical mapping are bacterial artificial chromosome (BAC) libraries. Whole genome sequencing projects of Arabidopsis [12], rice [2] and maize $[4,13,14]$ have all benefited from BAC-based physical maps to produce a scaffold structure for the construction of large, assembled chromosome sequences or pseudomolecules. Contigs 
constructed from BAC clones provide a backbone to which sequence contigs can be anchored. This is especially valuable in large and repetitive genomes where simple whole-genome shotgun sequencing usually leads large numbers of un-ordered sequence contigs.

To reduce complexity of the wheat genome analysis, individual chromosomes (for the largest chromosome 3B) or chromosome arms can be isolated through cytometric flow-sorting based on their individual 'flow karyotype' [15]. Ditelosomic chromosomespecific wheat lines are used to purify individual chromosome arms that can then be used for the production of chromosome-specific BAC libraries, survey sequencing and physical maps $[9,15,16,17]$. Chromosome $3 \mathrm{~B}$, the largest chromosome in wheat $(\sim 1 \mathrm{~Gb})$, was the first chromosome to be physically mapped. A chromosome 3Bspecific BAC library was produced from flow-sorted chromosomes which was then used for the development of the physical map of wheat chromosome 3B [18].

Flow-sorted chromosome arms were used recently for shotgun sequencing with Illumina technology in the framework of the International Wheat Genome Sequencing Consortium (IWGSC). The sequence assemblies of this so-called "whole chromosome survey sequencing" are available through the IWGSG website (wheatgenome.org). The assemblies resulted in hundreds of thousands of sequence contigs per chromosome arm. These sequence contigs are mostly un-ordered but, nevertheless, are expected to cover almost the complete gene space of the individual chromosome arms. A fraction of these sequences was ordered based on synteny using the chromosome zipper method [6]. Furthermore, the entire genome of wheat was recently surveyed by whole-genome shotgun sequencing. However, due to the large size and high repeat content of the genome, sequence assembly had to be limited on the gene-containing fraction [19].

BAC clones are typically digested with restriction enzymes which produces a specific fragment pattern for each BAC clone. This "fingerprint" is then used to find overlaps between BACs and arrange them into physical BAC contigs. Modern high information content fingerprinting (HICF) is currently using restriction enzymes that produce particularly diverse fingerprint patterns. FPC [20] has been the most popular fingerprint construction program for previous grass genome projects. However, the application of FPC is limited when dealing with complex genomes such as wheat because highly repetitive, chimeric or poorly fingerprinted "questionable" clones (Q-clones) can create false overlaps and thus wrongly assemble contigs (called Q-contigs [21]). To improve fingerprint contig assembly in highly repetitive genomes, the Linear Topological Contig (LTC) algorithm [21] was developed. It improves contig assembly by avoiding large branching structures from multiple neighbouring BAC connections, to create physical contigs with linear topology.

The available genomic sequence resources in wheat described above are complemented by over 1 million expressed sequence tags (ESTs) which are publicly available at NCBI (ncbi.nlm.nih.gov). These allow the characterisation of expressed genes as well as the assembly of so-called UniGene EST contigs. For wheat, a NimbleGen $12 \times 135 \mathrm{~K}$ microarray was designed containing 40,349 probes made from wheat UniGene sequences [22]. This microarray can be used as a proxy to associate gene content with BAC clones without having the complete sequence information: hybridising BAC pools to that microarray allows the rapid identification of BAC clones containing a homolog of a UniGene that is present on the microarray. However, the results have to be used with caution because hybridisation signals do not distinguish between orthologs, paralogs, pseudogenes or gene fragments. Nevertheless, this resource was successfully used for the construc- tion of a 3,000-locus transcriptome map for wheat chromosome 3B [22].

Low resolution physical mapping of genes has been done for several years on wheat lines which contain partial deletions of chromosome arms. This so-called "deletion-bin mapping" was done with wheat ESTs across all wheat chromosomes [23,24] and showed that genes were more frequently found in distal chromosomal regions than in proximal regions. Recent studies using transcriptome mapping [22,25] largely confirmed these findings but also indicated that about $75 \%$ of the genes on chromosome $3 \mathrm{~B}$ are located in small gene islands containing on average three genes [25], thereby supporting earlier studies that suggested clustering of genes in certain regions [26,27].

The short arm of chromosome $1 \mathrm{~A}$ is one of the smallest chromosome arms of wheat and was estimated through cytogenetic methods to have a size of $275 \mathrm{Mb}$ [15]. Chromosome arm 1AS is free of large C-banding regions that indicate regions of constitutive heterochromatin [28]. It contains the disease resistance genes Pm3 [29] and $\operatorname{Lr} 10$ [30] as well as gene underlying other important traits such as low molecular weight (LMW) glutenin genes [31] and the tiller inhibition gene (tin) [32]. Furthermore, it contains at least three families of resistance gene analogs (RGAs, [33]).

As member of the International Wheat Genome Sequencing Consortium, we have produced a physical map of the short-arm of chromosome 1A. This physical map was assembled through highinformation content fingerprinting (HICF) of a chromosome 1ASspecific BAC library using two different physical mapping algorithms and anchored to a chromosome lAS transcriptome map using high-throughput microarray hybridisation. Additionally, we produced an integrated sequence model that consists of genomic sequences that were ordered based on all available information resources. It comprises at total of $3.8 \mathrm{Mbp}$ of sequences and consists of BAC-end sequences and 1AS survey sequence contigs that were anchored to the BAC contigs through genetic mapping and NimbleGen hybridisation data. This highly integrated map can serve as a framework for future sequencing and map-based cloning studies.

\section{Methods}

\section{Purification of Chromosome Arm 1AS}

Aqueous suspensions of metaphase chromosomes were prepared from root tips of double ditelosomic line $(2 n=40+2 t 1 \mathrm{AS}+2-$ t1AL) of Triticum aestivum L. cv. 'Chinese Spring' according to Vrána et al. [34]. After staining by DAPI, the samples were analysed using FACSVantage flow sorter and 1AS arm was purified. The arm was sorted in batches of 200,000 into $320 \mu \mathrm{l}$ of $1.5 \times$ IB buffer [35], and a total of 8.85 million $1 \mathrm{AS}$ arms were collected. The identity and purity in sorted fractions was checked by FISH using probes for telomeric repeats and GAA repeats [36]. The purity of the sorted 1As fraction was 94\% (olomouc.ueb.cas.cz/dnalib/taacsplasha), and $6 \%$ of the collected DNA stemmed from other wheat chromosomes.

\section{Construction of 1AS-specific BAC Library}

The library (named TaaCsplAShA) was constructed as previously described [37]. High molecular weight DNA was partially digested with HindIII (New England Biolabs, Beverly, USA) and subjected to two rounds of size selection. DNA of particular size fractions was electro-eluted from the gel and ligated into pIndigoBAC-5 vector (Epicentre, Madison, USA). The recombinant vector was used to transform Escherichia coli DH10B competent cells (Invitrogen, Carlsbad, USA). The library was 
ordered into 384-well plates filled with freezing medium consisting of 2YT, $6.6 \%$ glycerol and $12.5 \mu \mathrm{g} / \mathrm{ml}$ Chloramphenicol and stored at $-80^{\circ} \mathrm{C}$. In order to estimate average insert size, $160 \mathrm{BAC}$ clones were randomly selected from both size fractions of the library and analyzed as previously described [36].

\section{Fingerprint Assembly and Minimum Tiling Path (MTP) Selection}

Fingerprinting of the chromosome 1AS-specific BAC library [15] was performed using a modified SNaPshot high-information content fingerprinting (HICF [38]) protocol used previously for creating hexaploid wheat physical maps [18]. Fingerprint background (vector, low signal and partially digested-related peaks) was removed using FPB [39] and contamination was removed using GenoProfiler [40].

Automated computational assembly of fingerprints was performed using FPC [19]. Standard physical map assembly procedures were followed based on previously described protocols from wheat physical mapping [18]: Initial assembly of 25,918 clones (incremental contig building) was performed at $1 \mathrm{E}-75$, then merging (single-to-end followed by end-to-end) was performed step-wise by increasing the cut-off ( $1 \mathrm{E}-5$ at each step) to a final cutoff of 1E-45. The DQer function was used after each merge to break up all contigs that contained more than $10 \%$ of questionable (Q) clones. The minimum tiling path (MTP) was selected with the FPC MTP module [41], which was then used to create threedimensional pools for marker screening [42] and BAC-end sequencing using Sanger ABI3730 sequencer. The 3D-pools were validated using the published chromosome $1 \mathrm{AS} \mathrm{cv}$. Chinese Spring-specific gene markers for Pm3 [29] and Lr10 [30].

Physical contigs and MTP BACs were screened using LTC [21] to identify problematic contigs, followed by a complete reassembly by LTC. Initial assembly was performed at a stringency of 1E-15, with Q-contigs (contigs displaying non-linear network structures) being separated at higher stringency (up to 1E-45). Network files of all Q-contigs were analysed using Pajek (vlado.fmf.uni-lj.si/pub/ networks/pajek/). Q-clones creating non-linear conformations were then fragmented and successive adaptive clustering algorithms were used to produce large contigs. Physical contigs containing at least 6 clones were kept for downstream analysis.

\section{Sequence Analysis}

BAC-end sequences were analysed through BLASTN and BLASTX searches against sequence databases. Triticeae-specific repetitive elements were searched against both nucleotide and protein subsets of the Triticeae-specific repeat database (TREP, wheat.pw.usda.gov/ITMI/Repeats/) to identify characterised transposable elements (TEs). Genes were identified by searching the Brachypodium distachyon (IBI2010) gene and tRNA, rDNA, mitochondrial and chloroplast DNA sequences (ncbi.nlm.nih.gov/ Genome). Closest Brachypodium homologs of Unigene sequences were identified by BLASTX against Brachypodium proteins (IBI2010). Hybridised Unigene probe matches were screened for TEs against TREP and searched against deletion bin-mapped 1AS-specific wheat ESTs [43]. All BLASTX searches were performed using BLASTX alignments with a $<1 \mathrm{E}-10$ E-value cut-off. Cut-off for BLASTN searches were alignments of at least 50 bp with $>80 \%$ identity and an E-value of $<1 \mathrm{E}-10$.

\section{Anchoring of Genetic Markers}

Genetic markers (Table 1) were gathered from published genetic maps contained in the marker repository GrainGenes (wheat.pw.usda.gov) based on their presence on chromosome 1AS.
Initial anchoring of physical contigs to maps was performed using standard PCR screening on MTP 3D-pools using published genetic markers (RFLP and SSRs). Additionally, EST/cDNAbased markers were designed based on low coverage 454 sequencing from flow-sorted chromosome 1AS [9]. If marker sequence data (e.g. primers or RFLP sequences) were available, these sequences were used in Blastn searches against BAC-end and anchored Illumina sequences (see below). For primers, we required perfect matches for anchoring, while we accepted matches with $>97 \%$ for RFLP sequences.

\section{Wheat NimbleGen $40 \mathrm{~K}$ UniGene Microarray Hybridisation}

Forty-nine DNA pools were generated from the MTP clones. The MTP was derived from FPC contigs which cover approximately $86 \%$ of chromosome arm 1AS (see results and discusison). The DNA pools were used for hybridisation on a custom $12 \times 135 \mathrm{~K}$ NimbleGen gene expression array (nimblegen.om/ produts/expression/index.html) containing 40,349 NCBI wheat UniGene EST clusters (ncbi.nlm.nih.gov/unigene). Hybridisation procedures were carried out as previously described [22] using identical $12 \times 135 \mathrm{~K}$ array specifications, but no dye-swap was performed. Normalisation of hybridisation data was performed using the $\mathrm{R}$ statistical package ( $\mathrm{r}$-project.org). Thresholds from single-dye hybridisation were calculated from the probe data against each pool to eliminate outliers. Genes hybridising to 3 probes were selected and median values were calculated for each gene. Genes with a ratio equal to 1 were eliminated. Box-plots were calculated for all other ratios to identify outliers and define threshold values. These threshold values were then used to remove outlier values for pools labelled with either cy3 or cy5 dyes. Values greater than the mean $(+/-$ standard deviation) for each gene and pool were extracted with previously identified thresholds [22]. To detect positive signals, two different methods were used, the socalled Mean $+x$ Standard deviation $(\mathrm{m}+\mathrm{xSD})$ method and t-tests, as described previously $[22,25]$. After data de-convolution, unique addresses were taken from the highest stringency of each method (2.4 for plates, 2.7 for rows, 2.8 for columns for $\mathrm{M}+\mathrm{xSD}$ and $\mathrm{p}$ value 0.01 for t-tests).

Hybridised UniGene probe matches were screened for TEs by Blast against the TREP database. They were also searched against deletion bin-mapped 1AS-specific wheat ESTs [38]. All Blastx searches were performed using a $<1 \mathrm{E}-10$ E-value cut-off. BLASTN searches were run using a 50 nucleotide alignment with $>80 \%$ identity and a $<1 \mathrm{E}-10$ E-value cut-off.

\section{Production of an Integrated Sequence Model from Illumina Survey Sequences}

Illumina sequence contigs for chromosome $1 \mathrm{AS}$ were obtained though the sequence repository of the International Wheat Genome Sequencing Consortium (wheat-urgi.versailles.inra.fr/ Seq-Repository). The 1AS survey sequence assembly consists of 187,490 contigs with a cumulative length of $178 \mathrm{Mbp}$. To anchor the sequence contigs to the physical BAC contigs, the Illumina contigs were used as queries in Blastn searches against BAC-end sequences of the $1 \mathrm{AS}$ minimum tiling path. Blast alignments of $>500 \mathrm{bp}$ and $>98 \%$ sequence identity were accepted for anchoring a sequence. The Illumina contigs were used without repeat masking. This was done to allow anchoring of possible gene sequences that are located next to repetitive sequences on an Illumina contig. The stringent criteria (>500 bp and $>98 \%$ sequence identity) used for the selection of Blast hits reduces the chance that repetitive sequences are wrongly anchored. 
Table 1. Genetic markers linked to the chromosome 1AS physical map.

\begin{tabular}{|c|c|c|c|c|c|c|c|c|c|c|}
\hline Marker & Zipper $^{1}$ & $\operatorname{TmGxG}^{2}$ & $\mathrm{TaBxT}^{3}$ & $\mathrm{TaAxF}^{4}$ & $\mathrm{TaNx} \mathbf{W}^{5}$ & $\mathrm{TaSxO}^{6}$ & $\mathrm{TaCxCS}^{7}$ & ConSSR $^{8}$ & Com $2004^{9}$ & ConsPos $^{10}$ \\
\hline bcd1434 & 18 & 5.1 & - & - & - & - & - & - & - & 5 \\
\hline psp2999(Pm3) & 28 & 4.8 & 3 & 1 & - & 14 & - & - & 12 & 7 \\
\hline whs179 & 29 & - & - & - & - & - & - & - & - & 10 \\
\hline gmw136 & 29 & - & - & - & - & - & - & - & 14 & 14 \\
\hline cfa2153 & 39 & - & - & 1 & - & 13.8 & 5.4 & - & 15 & 9 \\
\hline barc148 & 48 & - & - & - & 55 & 33.4 & - & 56.8 & - & 48 \\
\hline F640(Lr10) & 56 & - & - & - & - & - & - & - & - & 22 \\
\hline mwg2245b (Lr10) & 56 & - & - & - & - & - & - & 22 & - & 22 \\
\hline mag1884 & 88 & - & - & - & 22 & - & - & - & - & 22 \\
\hline gpw2005 & 122 & - & - & - & - & - & 27.3 & - & 31 & 29 \\
\hline wmc24 & 176 & - & - & - & 45 & 35 & - & 48.8 & 37 & 41 \\
\hline gpw2142 & 343 & - & - & - & - & 43.1 & - & - & 54 & 49 \\
\hline cfd58 & 452 & - & - & 52.9 & - & - & - & - & - & 53 \\
\hline psp3027 & 496 & - & - & - & - & - & - & - & 50 & 50 \\
\hline fba393 & 588 & - & - & - & - & - & 12.5 & - & 21 & 17 \\
\hline wmc286 & C & - & - & - & - & - & - & - & 45 & 45 \\
\hline
\end{tabular}

${ }^{1}$ Position of the closest gene in the reference zipper.

${ }^{2}$ TmGxG: T. monococcum G1777 ×G2528 [67].

${ }^{3}$ TaBxT: Banks $\times$ Banks+tin (Spielmeyer et al., unpublished).

${ }^{4} \mathrm{TaAxF}$ : Arina $\times$ Forno [68].

${ }^{5}$ TaNxW: Nanda2419 $\times$ Wangshuibai [69].

${ }^{6} \mathrm{TaS} \times \mathrm{O}:$ Syntethic $\times$ Opata [70].

${ }^{7} \mathrm{TaCxCS}$ : Courtot $\times$ Chinese Spring (Sourdille, unpublished)

${ }^{8}$ ConSSR: Consensus SSR [53].

${ }^{9}$ Com2004: Wheat-Composite 2004 (Appels et al., unpublished).

${ }^{10}$ ConsPos: Consensus position (approximate average of all $\mathrm{CM}$ positions for each marker).

Genetic positions (telomere to centromere) from different maps were taken from published genetic maps in GrainGenes (wheat.pw.usda.gov). The numbers in the individual marker fields indicate the $\mathrm{cM}$ position of the respective marker. Note that markers mapping to the same position in the reference zipper can have multiple different $\mathrm{CM}$ positions, depending on the map/population.

doi:10.1371/journal.pone.0080272.t001

Additionally, Illumina contigs were used in Blast searches against the 40,349 UniGenes that are represented on the NimbleGen microarray. Here, we used a cut-off of $200 \mathrm{bp}$ and $>90 \%$ sequence identity to anchor a sequence. We used less stringent criteria for UniGenes because the microarray represents genes from the entire wheat genome, which makes it very likely, that many signals come from paralogous genes. We considered the low stringency legitimate because the Illumina survey sequences specifically represent chromosome $1 \mathrm{AS}$, thereby reducing the probability that paralogs were wrongly anchored. However, this does not exclude the possibility that members of large gene families from within IAS are anchored incorrectly. Each Illumina contig was allowed to be anchored only in one place (i.e. the same sequence can not occur multiple times in the integrated sequence model).

\section{Data Deposition}

The chromosome IAS BAC library is available through the Centre National de Resources Génomiques Végétales (CNRGV), Toulouse, France (cnrgv.toulouse.inra.fr/) and the Institute of Experimental Botany, Olomouc, Czech Republic (http:// olomouc.ueb.cas.cz/dna-libraries/cereals). Physical mapping and marker data is available from the Unité de Recherche Génomique Info (URGI) at Institut National de la Recherche Agronomique (INRA), France (wheat-urgi.versailles.inra.fr/Projects/TriticeaeGenome2). The LTC BAC contig information can be accessed through the dedicated Gbrowse browser.(http://urgi.versailles. inra.fr/gb2/gbrowse/wheat_phys_pub/). The integrated sequence model was deposited at at the URGI sequence repository (http://wheat-urgi.versailles.inra.fr/Seq-Repository) in the 1A section. The sequence can also be obtained from the authors via FTP upon request.

\section{Results and Discussion}

\section{Chromosome 1AS BAC Fingerprinting and Selection of a Minimum Tiling Path}

The 1AS-specific library (TaaCsplAShA) consists of 31,104 clones with an average insert size of $111 \mathrm{~kb}$. Assuming a cytogenetically estimated chromosome arm size of $275 \mathrm{Mb}$ [15], the library provides a chromosome arm coverage of approximately 11.8x. High information content fingerprinting (HICF, [41,43]) yielded 25,918 high-quality fingerprints $(83.3 \%$ of the library). Initial fingerprint assembly using FPC resulted in 805 physical contigs with an average size of $294 \mathrm{~kb}$. The largest contig has a size of approximately 2,114 kb and is comprised of 211 BACs. The total assembly length is $236 \mathrm{Mb}$ corresponding to approximately $86 \%$ of the size of 1 AS (Table 2 ). A minimum tiling path (MTP, the minimum number of BAC clones necessary to cover all assembled contigs) of 3,414 BACs was selected from the $805 \mathrm{FPC}$ contigs (average of 4.25 BACs per contig). From this selected MTP, we designed 49 three-dimensional pools (plate, row and column) to allow efficient PCR screening. Additionally, the ends of all MTP BACs were sequenced (see below). 
Table 2. Comparison of the results of two physical map assembly algorithms used to assemble chromosome 1AS fingerprints, FPC and LTC.

\begin{tabular}{lll}
\hline & FPC & LTC \\
\hline Number of contigs & 805 & 505 \\
Contigs with more than 5 BACs & 631 & 394 \\
Assembly length [Mb] & 236 & 226 \\
Chromosome arm fraction & $85.8 \%$ & $82 \%$ \\
Maximum clones on a contig & 274 & 317 \\
Clones in contigs & 20,705 & 21,622 \\
Singletons & 5,213 & 4,296 \\
Contigs in N50 & 175 & 90 \\
Length of N50 contig [kb] & 466 & 798 \\
\hline
\end{tabular}

doi:10.1371/journal.pone.0080272.t002

\section{BAC Fingerprint Assembly Improvement using the Linear Topological Contig Algorithm}

The Linear Topological Contig (LTC) algorithm [21] became available after our initial FPC fingerprinting and MTP selection. Thus, we reassembled fingerprints with LTC to evaluate the accuracy of the FPC protocol. LTC was used to perform clone network analysis of FPC contigs and identify problematic contigs that did not conform to a linear topography (FPC_ctg212 is shown as an example in Figure 1). An initial assembly build on all 25,918 clones was run using LTC to establish physical contigs at a Sulston score of 1E-15 using adaptive clustering methods. 340 physical contigs were constructed and Q-contigs were identified that contained non-linear conformations or Q-clones that were not supported by other, parallel clones (i.e. putative chimeric clones). Many Q-clones causing false connections between clones in Qcontigs were identified to be from the same fingerprint sequencing plate, indicating that well-to-well contamination was a major cause of creating non-linear assembled contigs. Similar problems were previously reported when assembling a FPC map in maize [44]. Q-contigs were then rebuilt with an increased stringency up to $1 \mathrm{E}$ 50 in order to disperse the non-linear clusters. After breaking up Q-contigs, the LTC assembly consisted of a total of 505 contigs (Table 2). After all contigs showed linear clone patterns, small contigs containing five clones or less and no marker information (see below) were removed from the assembly as they did not provide any more meaningful information. This resulted in a more accurate final dataset of 394 LTC contigs which was used for subsequent analyses (Table 2).

\section{Comparison of FPC and LTC Assemblies}

The characteristics of the 394 LTC linear contigs were compared to those of the 805 contigs produced with FPC (Table 3). Overall, the LTC assembly included more BAC clones than the FPC assembly (21,622 vs. 20,705), leaving only 4,296 singletons compared to the 5,213 singletons left by FPC. The N50 number of contigs (the least number of contigs that contain $50 \%$ of the total assembly) fell from 175 to 90 and the length of the N50 contig increased from $479 \mathrm{~kb}$ to $798 \mathrm{~kb}$ using LTC (Table 3).

To assess the differences between the LTG and the FPG assemblies, we designed software that compares each LTG contig with the corresponding FPC contigs. As the examples in Figure 2a and $2 \mathrm{~b}$ show, the lower number of LTC contigs and their larger size is mainly due to the merging of multiple FPC contigs into one LTC contig. In total, 208 LTC contigs are fusion products of 472 FPC contigs. In all except 6 cases, the fused FPC contigs actually overlapped but were not viewed significant by FPC. On average the merged FPG contigs overlapped by approximately $57 \mathrm{~kb}$ (Figure 2c). In 71 cases, the merging was done by including additional clones that were initially marked as singletons in the FPC assembly. There, the average overlap of the merged FPC contigs was $42 \mathrm{~kb}$ (Figure 2c). In six cases, a gap between contigs was closed entirely with FPC singletons (example in Figure 2b). These data indicate that the LTC program was more successful in producing larger, more integrated assemblies.

The reassembly of fingerprints using LTC and the comparison to the results from FPC highlighted two major problems of BAC fingerprinting in large and repetitive genomes. First, despite the use of cleaning software [34], well-to-well contamination during the HICF fingerprint sequencing can lead to many Q-clones, and in turn, non-linear Q-contigs. The fact that LTG removed these from the assembly is probably the reason why the 394 LTC contigs (despite an overall larger size of the contigs) cover only approximately $226 \mathrm{Mb}$ (82\% of the 1AS chromosome arm) while FPG contigs reached a $86 \%$ coverage of 1 AS (Table 2). Second, the complexity of the wheat sequence limited the use of the standard physical mapping software FPC, because non-linear contigs were produced already at the initial contig construction, creating a faulty base for the map.

\section{The 1AS Backbone Consists of LTC BAC Contigs Complemented with FPC Clones}

The final dataset of 394 LTC BAC contigs represent over $80 \%$ of the backbone of the 1AS physical map (Table 3). However, these LTC contigs do not include all BACs, which were part of the minimum tiling path produced by FPC. These excluded BACs contain potentially useful information as they were used for the NimbleGen hybridisation (see below) as well as end-sequenced. To not lose this information, we added to the above dataset of 610 BAC clones that were excluded from the LTC assembly but were part of the minimum tiling path of the FPC assembly. Of these 610 BACs, 144 were singletons while the rest were arranged in 177 small contigs of 2-7 BACs. Thus, the final backbone of the 1AS physical map consists of 394 LTC contigs with a cumulative size of $205 \mathrm{Mbp}$ and $321 \mathrm{FPC}$ BAC contigs or singletons that cover $50 \mathrm{Mbp}$ (Table 3). The total cumulative size of all BAC contigs is 255.4 Mbp, corresponding to $92,8 \%$ of chromosome $1 \mathrm{AS}$ (Table 3).

In summary, we propose the use of both, LTC and FPC software. LTC overall performed better than FPC on IAS BAC fingerprints, resulting in larger and more robust contigs. However, we also considered it important to include BAC and BAC contigs that were leftover from the initial FPC assembly. Although this leftover FPC dataset consists of BAC singletons or very short contigs, its inclusion nevertheless increased the overall coverage of the lAS chromosome arm from $82 \%$ to almost $99 \%$. Because these additional FPC BAC contigs have an overall low coverage and are therefore less robust than the FPG contigs. Thus, they were flagged in the final backbone with the prefix "fpc".

\section{Sequence Composition of Chromosome 1AS Inferred from MTP BAC-end Sequencing}

BAC-end sequencing was performed on each of the 3,414 MTP BACs producing 6,687 sequences with an average size of $703 \mathrm{bp}$. The cumulative length of all BAC end sequences (BES) was 


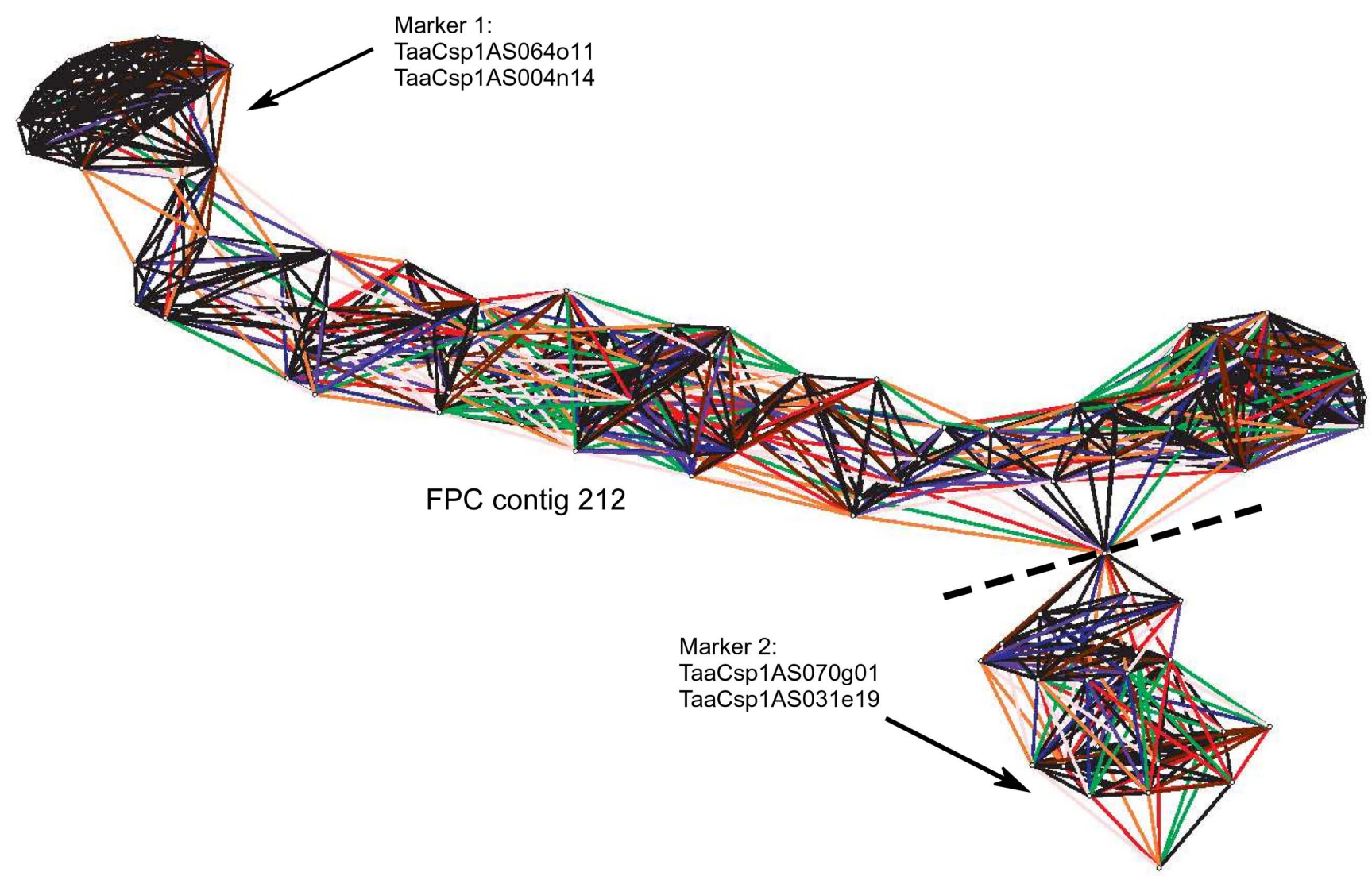

Figure 1. Pajek analysis of BAC contigs from chromosome 1 AS assembled with the FPC software. Repetitive BAC clones within the BAC fingerprints were problematic for the FPC assembly, leading to non-linear contig patterns. The reassembly of fingerprints using the LTC assembly program resolved non-linear contigs. The dashed line indicates where the non-linear contig was cut into two contig segments, because the two segments are only connected by a single BAC clone (indicated by all connections converging in one point.

doi:10.1371/journal.pone.0080272.g001

$4,704 \mathrm{~kb}(1.7 \%$ of the $1 \mathrm{AS}$ chromosome arm). These sequences allowed the quantitative study of the chromosome arm sequence composition. A total of 180 sequences $(2.6 \%)$ had homology with Brachypodium genes, 42 of which had their closest homologs in the lAS syntenic region in the Brachypodium genome. These BAC ends were used to anchor BAC contigs to the Brachypodium reference

Table 3. Final composition of the BAC clone backbone of the wheat 1 AS physical map.

\begin{tabular}{ll}
\hline & \\
\hline LTC contigs with >5 BACs & 394 \\
BACs in LTC contigs & 21,255 \\
Total size of LTC contigs [Mbp] & 226 \\
Included FPC contigs & 321 \\
BACs in FPC contigs & 610 \\
Total size of FPC contigs [Mbp] & 48 \\
Total number of contigs & 715 \\
Total BACs included & 21,865 \\
Total size of the backbone [Mbp] & 274 \\
Total coverage of 1AS [\%] & 99 \\
\hline
\end{tabular}

The backbone consist mainly of contigs assembled with the LTC software (Frenkel et al., 2010) and is complemented with BACs from an earlier assembly with the FPC software (Soderlund et al., 1997, Table 1).

doi:10.1371/journal.pone.0080272.t003 zipper (see below). As in previous surveys of wheat BES [11,45], a majority of sequences were identified as TEs, with $81.7 \%$ of the total DNA matching known elements $(77.7 \%)$ from the TREP database, or novel TEs $(4.0 \%)$ identified using protein matches to PTREP. Ribosomal and organellar DNA were present only in minuscule amounts and a total of $16.75 \%$ of all BES sequences could not be classified based on the databases used (Figure 3a).

The vast majority of TE sequences corresponded to elements of the Gypsy superfamily ( $42 \%$ of all BES). Copia and CACTA elements followed a distant second and third with $13.4 \%$ and $4.4 \%$ of all BES, respectively (Figure $3 \mathrm{~b}$ ). All other superfamilies, except for LINEs with $1.5 \%$, contributed less than $0.2 \%$ to all BES. A total of $25.7 \mathrm{~kb}(0.54 \%$ of all BES) matched the RLG_Cereba elements which are Gypsy retrotransposons that are highly specific for centromeres $[46,47]$.

Interestingly, the composition of $1 \mathrm{AS}$ BES sequences was somewhat unexpected with regard to individual TE families. The most abundant TE family was the Gypsy family Fatima, which contributed over 8\% to the total BES (Figure 3c). Similar findings were made on wheat chromosome $3 \mathrm{~B}$ where Fatima was also the most abundant element [9]. In previous genome surveys of diploid Triticeae species, Copia elements of the Angela/BARE1 family were clearly the most abundant with more than $10-12 \%$ of the whole genomic sequence [48-52]. In contrast, in our dataset, Angela/ BARE1 only contributed $4.16 \%$ to the BES. This could indicate that the TE composition of the hexaploid wheat genome differs from that of diploid Triticeae. Alternatively, it is possible that the 
a.

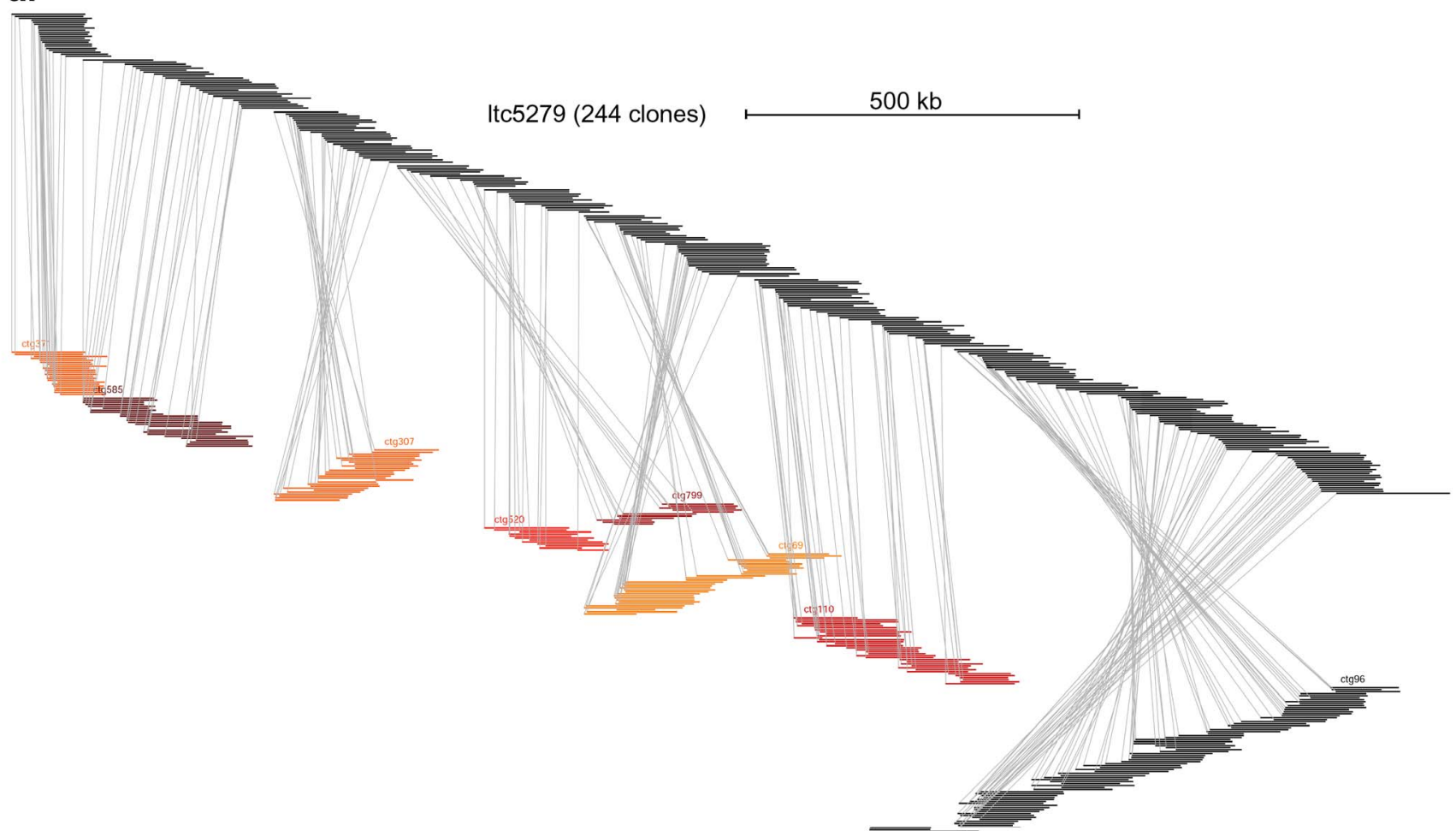

b.

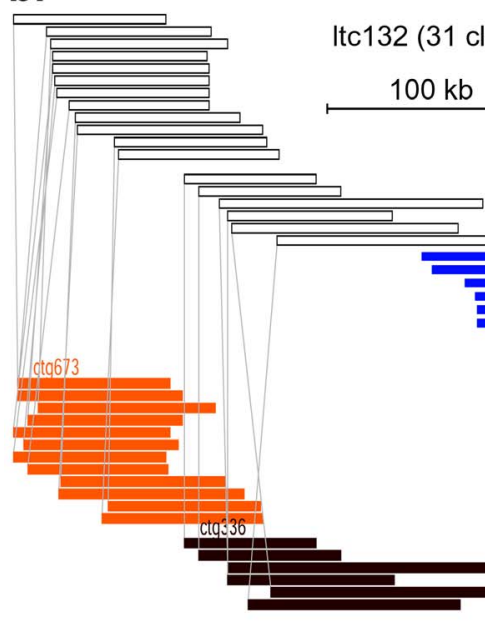

C.

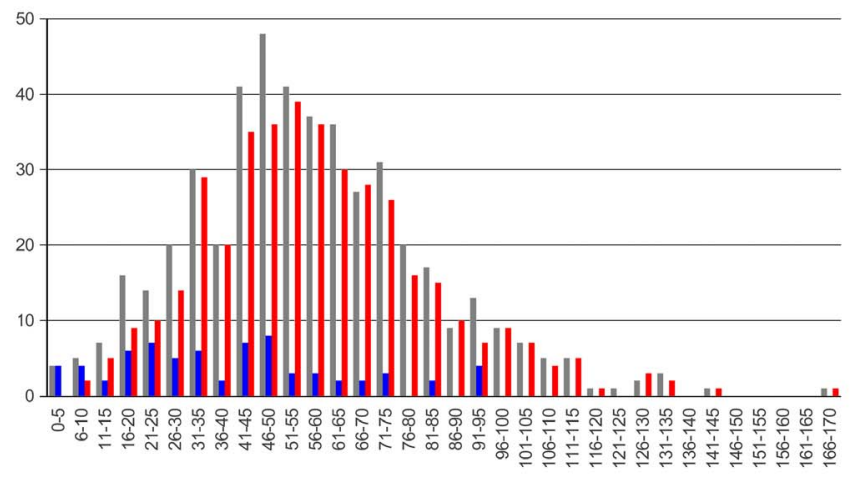

- All overlaps of merged FPC contigs

- Overlaps merged with additional singletons

- Overlaps merged without singletons

Figure 2. Comparison of LTC and FPC assemblies. a. The BAC clones constituting the contig Itc5279 are depicted at the top. Underneath, FPC contigs which cover corresponding regions are displayed. Gray lines connect the start points of corresponding BACs. Contig Itc5279 (approximately $2,161 \mathrm{~kb}$ in size) which is the fusion product of eight smaller FPC contigs. Overall, the relative positions of BAC clones within LTC and FPC contigs are very similar. b. Example of three small FPC contigs which are merged into one LTC contig (Itc132). This LTC contig also includes BACs which were singletons in the FPC assembly (blue). Note that in $\mathbf{a}$ and $\mathbf{b}$ the scales are different. c. Size distribution of overlaps of FPC contigs which were merged in the LTC assembly. The $x$-axis indicates the size range of overlaps of two FPC clones that were merged by LTC. The y-axis shows how many cases were identified in each size range. The gray series shows the size distribution of all overlaps. The blue series shows only those cases where additional singletons were included to merge FPC contigs while the red series shows the cases where no additional clones were used for the merging. doi:10.1371/journal.pone.0080272.g002

BAC MTP does not cover the repetitive fraction of the chromosome arm evenly.

\section{Construction of a 1S Reference Zipper from Brachypodium, Rice and Sorghum Genes}

Because only relatively few genetic markers were available which could be used to arrange BAC contigs into their correct physical order, we mostly used synteny (i.e. conserved order of genes) between Triticeae and sequenced model grass genomes to anchor BAC contigs. The region syntenic to wheat chromosome 1AS is located in the central part of Brachypodium chromosome 2 between genes Bradi2g30410 and Bradi2g40150 [5]. As wheat contains many TEs, we chose very stringent criteria for gene identification. First, we screened all 26,191 annotated Brachypodium 
a.

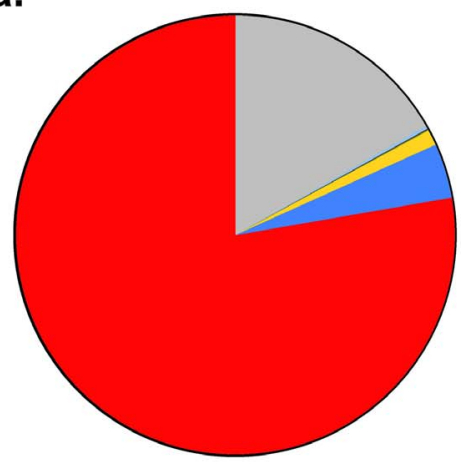

Known TE families Novel TE families

Brachypodium genes

- Ribosomal DNA

- Chloroplast DNA

Mitochondrial DNA

Not classified

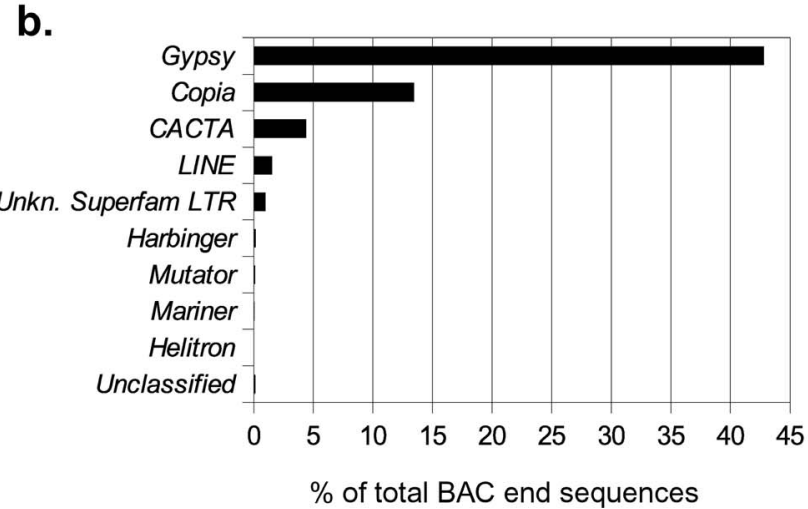

C.

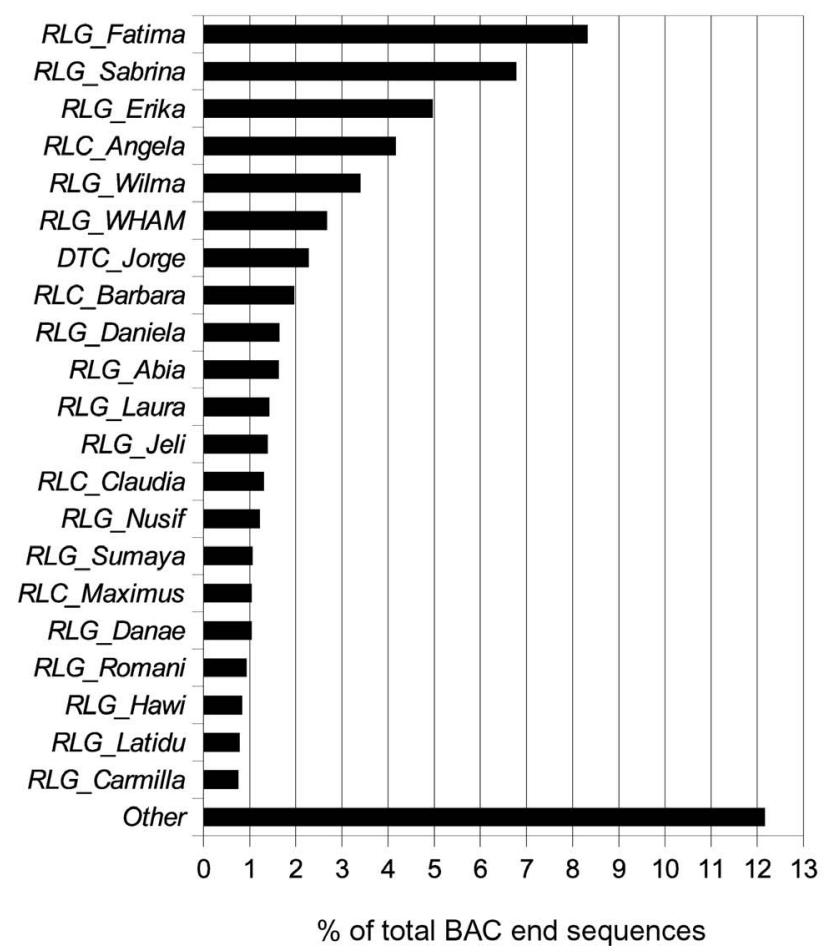

Figure 3. Sequence composition of minimum tiling path BAC-end sequences from chromosome 1AS. a. Pie chart indicating the composition of chromosome 1AS MTP BAC-ends. b. and c. Composition of the TE fraction. b. Composition of TE superfamilies. c. Composition of the TE fraction broken down into TE families. The three-letter code preceding the family name is according to the TE classification system proposed by Wicker et al., 2007.

doi:10.1371/journal.pone.0080272.g003

genes for TE sequences to identify TEs that were wrongly annotated as genes. The 732 sequences that showed homology to TEs were removed from the Brachypodium gene set. Second, we used all the TE-filtered Brachypodium CDS for BLASTN searches against the rice and sorghum genomes and only used genes that have homologs at the DNA level in Brachypodium, rice and Sorghum. This was done to minimise the number of annotation artefacts. A total of 21,059 Brachypodium genes have homologs in both rice and sorghum.

Using the above described criteria, Brachypodium contains 600 genes which fulfilled our criteria in the $1 \mathrm{~S}$ syntenic region. To account for genes that might have been moved out of this region in Brachypodium, we added ("zipped in") those Brachypodium genes whose homologs are present in the group 1 syntenic region in both rice and sorghum (this principle is described in more detail by Mayer et al. [7]). The final $1 \mathrm{~S}$ reference zipper contains 749 genes. To facilitate work with the reference zipper, the genes were numbered consecutively BdWlg-1 through BdWlg-749, with BdWlg-1 corresponding to the telomeric end of $1 \mathrm{AS}$ and BdWlg749 to the centromere. We refer to these numbers whenever we use the term "zipper position" or "zipper gene" hereafter in the manuscript.

Compared to rice and Sorghum, Brachypodium contains an inversion between zipper position 127 (Bradi2g38720) and 280 (Bradi2g37100). As genetic mapping indicated, this inversion is specific to Brachypodium and is not found in Triticeae (our own unpublished data). Thus, for our analyses, we inverted the order of all genes between 127 and 280 in the Brachypodium reference zipper.
Estimate of Gene Content of 1AS Chromosome Deletion Bins

Three chromosome deletion lines from wheat are available which lack different segments of $1 \mathrm{AS}$ (i.e. deletion bins). Bin 1AS0.86-1.00 corresponds approximately to the telomeric $14 \%$ of 1AS, bin $1 \mathrm{AS}-0.47-0.86$ to the region of $47 \%-86 \%$ of the length of $1 \mathrm{AS}$ and 1ASC- 0.47 corresponds to the centromeric $47 \%$ of 1AS. A total of 383 1AS-specific ESTs were previously mapped to the individual bins [23,24], 196 to the telomeric bin, 171 to the central and 16 to the centromeric bin (Table 4).

We used all bin-mapped ESTs in BLASTN searches against the Brachypodium reference zipper in order to estimate the total number of genes that are located in each of the three bins. A total of 120 ESTs showed homology at the DNA level to Brachypodium zipper genes. Often, multiple ESTs mapped to same gene and consequently, the $120 \mathrm{ESTs}$ actually only represent 67 different genes (Figure 4). Nevertheless, ESTs from the telomeric and the central bin clearly mapped to separate regions of the Brachypodium reference zipper (Figure 4), with few exceptions, which most likely represent non-colinear genes (see below). The boundary between the telomeric and the central bin can be narrowed down to a relatively small region approximately at zipper gene 200 (Figure 4).

The boundary between the central and the centromeric bin is less clear, as only two ESTs from the centromeric bin had matches on the Brachypodium reference zipper and both mapped to the same gene which is located near the telomeric end of the reference zipper (Figure 4). We therefore defined the centromeric bin by the absence of EST matches and the boundary between the central 
Table 4. Deletion bin-mapped wheat ESTs on wheat chromosome arm 1 AS and estimates of gene density within the bins.

\begin{tabular}{llllll}
\hline & & & & & \\
\hline Deletion bin & $\begin{array}{l}\text { size } \\
\text { [Mbp] }\end{array}$ & ESTs $^{\mathbf{a}}$ & $\begin{array}{l}\text { Zipper } \\
\text { hits }^{\mathbf{b}}\end{array}$ & $\begin{array}{l}\text { Zipper } \\
\text { genes }^{\mathbf{c}}\end{array}$ & $\begin{array}{l}\text { gene } \\
\text { density }^{\mathbf{d}}\end{array}$ \\
\hline 1AS-0.86-1.00 & 39 & 196 & 25 & $1-200$ & 5.12 \\
1AS-0.47-0.86 & 107 & 171 & 41 & $200-650$ & 4.20 \\
1ASC-0.47 & 129 & 16 & 1 & $650-749$ & 0.77 \\
Total & 275 & 383 & 67 & $1-749$ & 2.72 \\
\hline
\end{tabular}

${ }^{\mathrm{a}}$ Number of wheat ESTs mapped specifically to respective deletion Bin (Sorrels et al., 2003).

${ }^{\mathrm{b}}$ Number of genes in the Brachypodium 1S reference zipper which have homology at the nucleotide level to bin-mapped ESTs.

${ }^{c}$ Approximate range of zipper genes corresponding to wheat deletion bin.

dApproximate gene density, assuming the same number of genes for wheat as in the 1AS syntenic region in Brachypodium.

doi:10.1371/journal.pone.0080272.t004

and the centromeric bin to lie approximately at zipper gene 650 (Figure 4).

Assuming that the number of genes on IAS is similar to that in the Brachypodium reference zipper, we estimate the telomeric bin to contain approximately 200 genes (zipper positions 1 through 200) corresponding to a gene density of about 5.1 genes per Mbp. The central bin probably contains approximately 450 genes (zipper positions 201 through 650) and thus has a density of 4.2 genes per Mbp while the centromeric bin contains approximately 100 genes (zipper positions 651 through 749 ) at a gene density of 0.77 genes per Mbp. These estimates should be taken with caution as they only refer to putative syntenic genes and do not include nonsyntenic ones. Indeed, recent low-coverage survey sequencing of chromosome 1AS indicated the presence of a almost 1,000 nonsyntenic gene sequences. However, it is suspected that many of these may be gene fragments or pseudogenes [9].

\section{Level 1 Anchoring: Assigning Genes to BAC Contigs Through NimbleGen Array Hybridisation}

Three levels of anchoring were used to construct the integrated lAS physical map (Figure 5). At the most basic level (level 1, Figure 5) we compiled information from different sources to assign genes or information on gene homologs to individual BAC contigs. First, we hybridised the 49 chromosome 1AS MTP pools to a NimbleGen $12 \times 135$ K microarray containing 40,349 UniGene EST clusters. After de-convolution of the data, 647 hybridisation probes with matches to MTP BAC clones were identified. Of these 647 UniGene sequences, 389 had homology to Brachypodium genes (Figure 5, procedure A). Because some UniGenes cover different regions of the same gene, the 389 probes actually represent only 323 different genes. Of these, 154 have homologs in the reference zipper (Table 5). Here, it should be noted that we use the term "gene sequence" or "gene" simply meaning that the sequence shows homology to a known gene either at the DNA or at the protein level. However, we do not claim that all of the identified gene sequences are intact functional genes.

Overall 272 BAC contigs were identified which gave specific signals to hybridisation probes. The contigs hybridised with between 1 and 16 probes, but most hybridised with only one or two probes (Figure S1). A total of 113 contigs contained probes that have homologs in the Brachypodium reference zipper. These were subsequently used to anchor BAC contigs based on synteny to Brachypodium, rice and Sorghum (see below).
A total of 258 UniGenes have no Brachypodium homologs. One possible explanation is that these UniGene sequences represent genes which do not encode proteins or genes which are specific to wheat. Alternatively, it is possible that the UniGene sequence was derived from untranslated regions of the gene transcript.

\section{Level 1 Anchoring: Using BAC End and Illumina Chromosome Survey Sequences}

To test whether some of the 258 UniGenes without Brachypodium homologs could be derived from untranslated parts of transcripts, we used the 258 sequences in BLASTN searches against Illumina contigs from IWGSC chromosome survey sequencing of flow sorted wheat chromosome arms. We searched for cases where we find a homolog of a Brachypodium gene and a homolog of the UniGenes gene sequence next to it. These could represent cases where the UniGene represents untranslated regions of a gene. We identified 61 such cases. In 26 of them, the CDS neighbouring the UniGene had homology to a gene on the Brachypodium reference zipper (Figure 5, procedure B).

We also used MTP BAC end sequences to anchor genes to BAC contigs (Figure 5, procedure $\mathrm{C}$ ). As described above, we identified 180 BAC end sequences that have homology to Brachypodium genes, 42 of them have their closest Brachypodium homolog in the $1 \mathrm{~S}$ reference zipper. We also searched the IWGSC Illumina sequence contigs for perfect matches to $1 \mathrm{AS}$ BAC end sequences (see methods). The goal was the identification of gene-containing Illumina sequence contigs that could be anchored to BAC end sequences. With this approach, we were able to anchor additional 117 gene-containing sequences to BAC contigs, 32 of these genes have their best homologs in the Brachypodium 1S reference zipper (Figure 5, procedure D).

In summary, level 1 anchoring allowed us to assign a total of 755 gene sequences to 1 AS BAC contigs, 254 of them have homologs in the Brachypodium 1S reference zipper (Table 5) and were used subsequently in the synteny-based anchoring (level 2 anchoring).

\section{Level 2 Anchoring: Arranging BAC Contigs based on Synteny with Model Grasses}

The 254 genes that have homologs in the $1 \mathrm{~S}$ syntenic region in Brachypodium, rice and/or sorghum were used to order BAC contigs based on synteny to the three model grasses (i.e. BAC contigs containing these genes were arranged in the same order as the genes in the reference zipper). If a BAC contig contained multiple homologs to zipper genes, priority was given to the gene with the lower zipper number. For example, BAC contig LTC1712 contains homologs of Brachypodium genes Bradi2g39600 (zipper position 56) and Bradi2g39520 (zipper position 61). The contig was therefore anchored to zipper position 56 . We are aware that this could contribute to some contigs being placed in the wrong location.

In a side project, we tested whether PCR probes designed based on wheat EST sequences can be used to identify BAC clones carrying those genes. Because centromeric regions are notoriously difficult to work with we focused this effort on genes that were expected to lie in the proximal region of $1 \mathrm{AS}$ based on synteny. We designed PGR primers for 20 genes and were successful in identifying unique BAC addresses for four of the genes (Bradi2g30480, Bradi2g30850, Bradi2g31900 and Bradi2g32220), which map to positions $657,670,730$ and 744 of the reference zipper, respectively.

In total, we were able to anchor 158 BAG contigs to the reference zipper. The 158 contigs contain in total 580 gene 


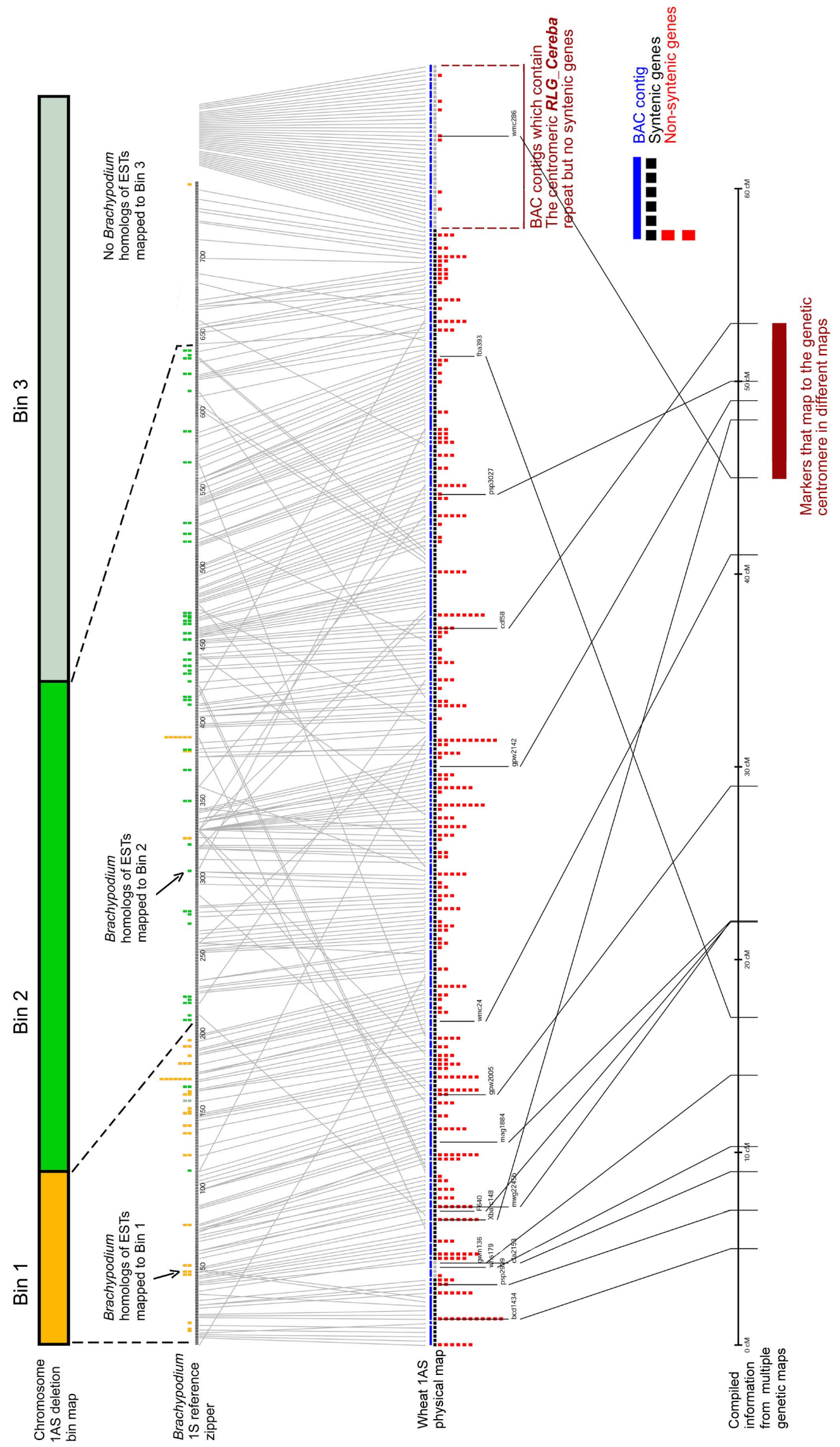


Figure 4. Physical map of wheat chromosome 1AS. The figure integrates multiple sequence resources. a. Chromosome 1 AS deletion bin map with the three bins shown in (yellow, green and gray). ESTs from the three deletion bins which were mapped to Brachypodium reference zipper genes are indicated with boxes with colour of the corresponding bin. If more than one EST mapped to the same Brachypodium gene, the boxes were stacked on top of each other. This information was used to estimate the boundaries of each deletion bin in the Brachypodium reference zipper (dashed lines). b. Brachypodium reference zipper. c. Physical map of the 1AS chromosme arm. BAC contigs are symbolised with blue lines (see enlarged legend at the right). The length of the line reflects the number of putative syntenic genes found on the contig, not its physical size. Syntenic genes are also symbolised by black boxes. The number of non-syntenic genes for each contig is indicated with a stack of red boxes. Grey boxes indicate place holders for contigs that contained no syntenic genes but were anchored by means other than synteny (e.g. genetic markers of centromere-specific repeats. d. Published genetic markers from chromosome 1AS that were used to deduce an estimated genetic map (marker and map names and genetic distances are detailed in Table 1).

doi:10.1371/journal.pone.0080272.g004

sequences, the 254 syntenic ones that were used for anchoring plus 326 that were non-syntenic. These numbers agree with previous findings that indicated that approximately two-thirds of the genes on $1 \mathrm{AS}$ are non-syntenic [8]. However, from the available data, we can not determine if these genic sequences represent functional genes or pseudogenes. The 158 anchored BAC contigs have a

Level 1 anchoring: Assigning genes to BAC contigs

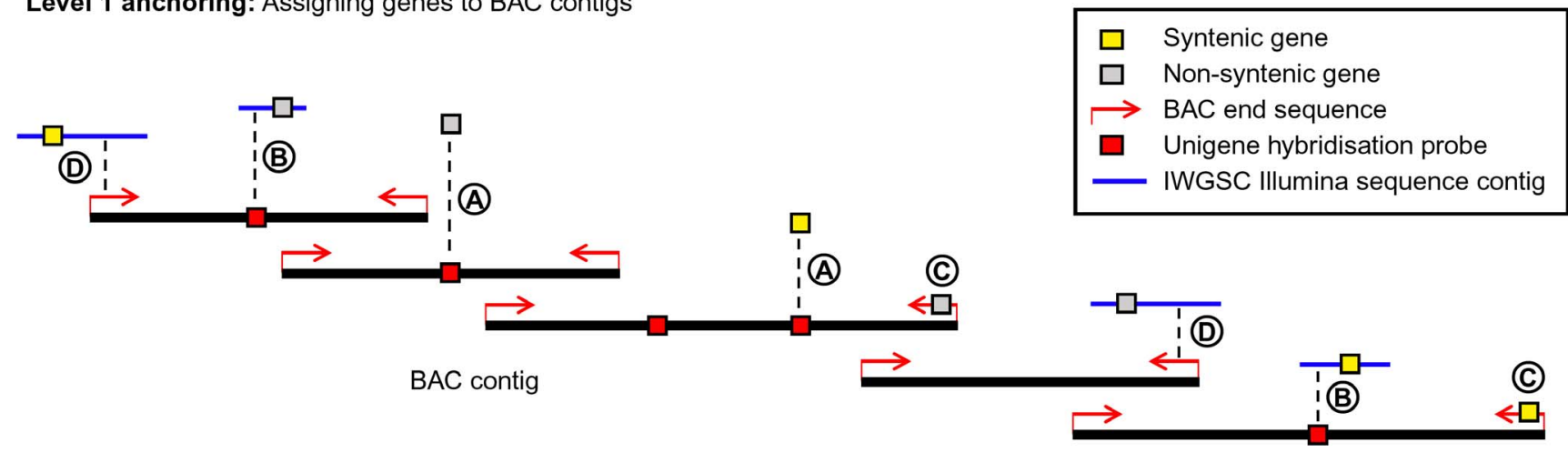

Level 2 anchoring: Ordering BAC contigs based on synteny

Reference zipper combining genes from the $1 \mathrm{AS}$ syntenic region from Brachypodium, rice and sorghum
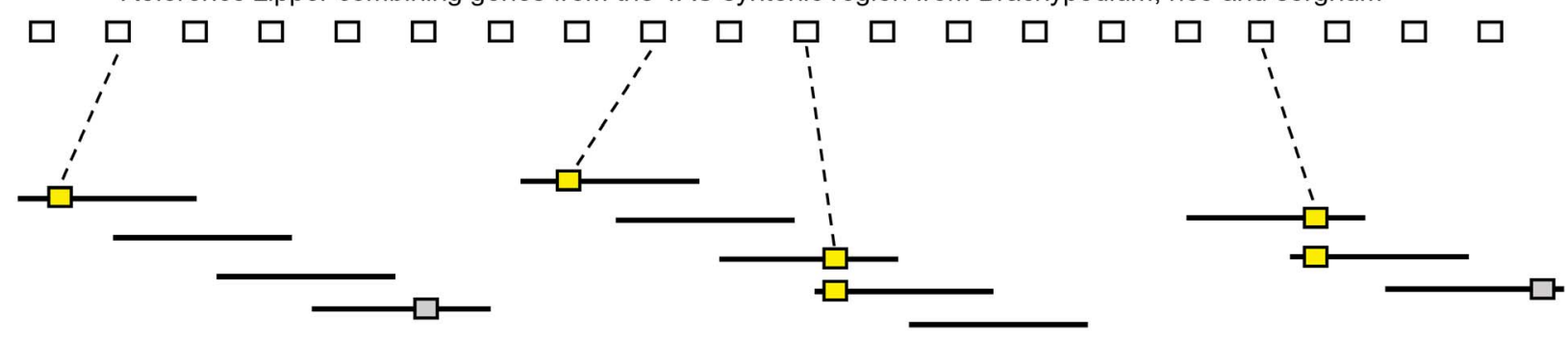

Level 3 anchoring: Anchoring BAC contigs to genetic maps

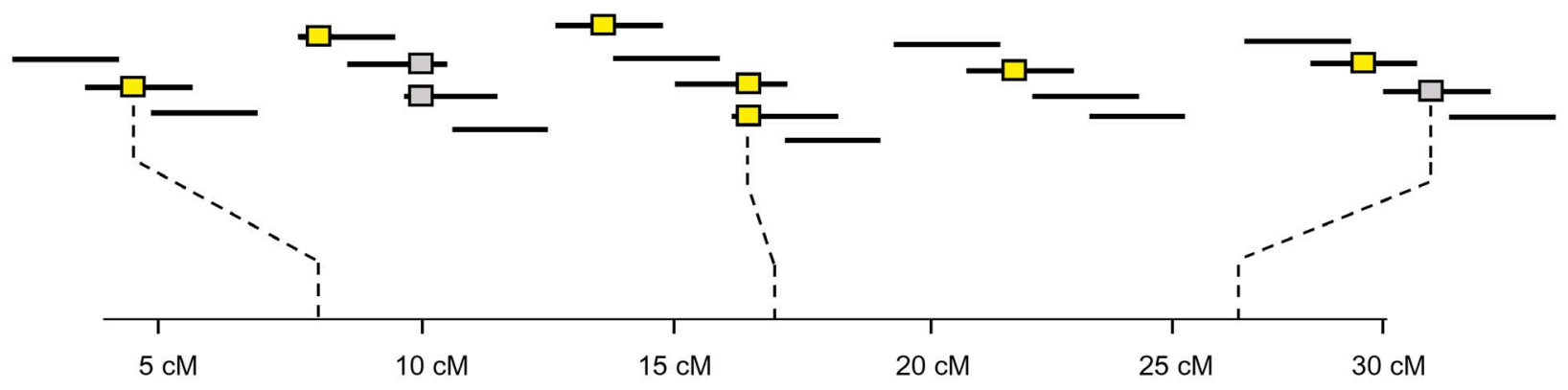

Figure 5. The three levels of anchoring used in the construction of the chromosome 1 AS physical map. On level 1, genes were anchored to physical BAC contigs using positive hybridisation probe matches, BAC-end sequences and Illumina contigs. Individual anchoring procedures are indicated by capital letters in circles and described in the text. For level 2 anchoring, all BAC contigs which contain genes which have their homologs in the 1AS syntenic region of Brachypodium, rice or sorghum were anchored to the reference zipper. This means that the order of genes in wheat was assumed to be the same as in Brachypodium, rice or sorghum. In the final step (level 3), data from genetic markers were used to anchor BAC contigs to previously published genetic maps.

doi:10.1371/journal.pone.0080272.g005 
Table 5. Numbers of genes which were assigned to 1 AS BAC contigs during level 1 anchoring.

\begin{tabular}{lll}
\hline Approach & Total genes & Zipper $^{\mathbf{a}}$ \\
\hline Hybridisation & 389 & 154 \\
BAC-ends & 180 & 32 \\
Illumina/BAC-end & 117 & 42 \\
Illumina/unigene & 61 & 26 \\
PCR & 0 & 4 \\
Total & 747 & 254 \\
\hline aGenes that have homologs in the 1 AS reference zipper and which could be \\
$\begin{array}{l}\text { used for level 2 anchoring. } \\
\text { doi:10.1371/journal.pone.0080272.t005 }\end{array}$
\end{tabular}

cumulative size of $102 \mathrm{Mbp}$, therefore representing approximately $37 \%$ of the $1 \mathrm{AS}$ chromosme arm (Table 6$)$.

\section{Level 2 Anchoring: Identification of Putative Centromeric BAC Contigs}

A total of 26 BAC contigs did not contain homologs of $1 \mathrm{AS}$ syntenic genes but had BAC-end sequences that contain the $R L G \_$Cereba LTR retrotransposon which is highly specific for centromeres in Triticeae genomes [46,47]. Thus, these 26 contigs were added to the physical map as a separate centromere segment. As the only criterion for the placement was the presence of $R L G \_$Cereba elements, the order of the BAC contigs inside the centromere segment is arbitrary. The $26 \mathrm{BAC}$ contigs have a cumulative size of $10.7 \mathrm{Mbp}$ which corresponds to approximately $4 \%$ of the size of the $1 \mathrm{AS}$ chromosome arm. A total of 40 genic sequences were identified in these 26 BAC contigs, all of which are

Table 6. Statistics on level 2 anchoring of BAC contigs in the 1 AS physical map.

\begin{tabular}{|c|c|}
\hline \multicolumn{2}{|l|}{ Non-centromeric segment } \\
\hline Non-centromeric contigs & 158 \\
\hline Non-centromeric contigs size [Mbp] & 102.1 \\
\hline 1AS fraction covered [\%] & 37 \\
\hline Syntenic genes ${ }^{a}$ & 254 \\
\hline Non-syntenic genes ${ }^{\mathrm{b}}$ & 326 \\
\hline \multicolumn{2}{|l|}{ Centromeric segment } \\
\hline Centromeric contigs & 26 \\
\hline Centromeric contigs size [Mbp] & 10.7 \\
\hline 1AS fraction covered [\%] & 4 \\
\hline Genes on centromeric contigs & 40 \\
\hline \multicolumn{2}{|l|}{ Total 1 AS map } \\
\hline Total achored contigs & 185 \\
\hline Total contig size [Mbp] & 112.8 \\
\hline Total 1AS fraction covered [\%] & 41 \\
\hline total genes & 620 \\
\hline \multicolumn{2}{|c|}{$\begin{array}{l}{ }^{\mathrm{a} G e n e s} \text { which have their closest homolog in the } 1 \mathrm{AS} \text { syntenic region of } \\
\text { Brachypodium, rice and sorghum. } \\
{ }^{\mathrm{b}} \text { Genes which have their closest homolog outside the } 1 \mathrm{AS} \text { syntenic region of } \\
\text { Brachypodium, rice and sorghum. } \\
\text { doi:10.1371/journal.pone. } 0080272 . \mathrm{t} 006\end{array}$} \\
\hline
\end{tabular}

non-syntenic (otherwise they would have been used for syntenybased anchoring).

\section{Level 2 Anchored BAC Contigs Cover $41 \%$ of the 1 AS Chromosome Arm}

The 158 BAC contigs that were anchored through synteny plus the 27 putative centromeric BAC clones have a combined length of $112.8 \mathrm{Mbp}$, thus covering approximately $41 \%$ of the $1 \mathrm{AS}$ chromosome arm (Table 6). In total $44.1 \%$ of the complete assembly was anchored to the map. Anchored physical contigs contain between 1 and 17 genes, with one contig (LTC-4568) containing the maximum. LTC-4568 also has the most syntenic (9) and non-syntenic genes (8). Eleven syntenic genes were duplicated (i.e. homologs to the same Brachypodium gene were found on more than one physical contig).

In barley, synteny-based anchoring has been shown to reflect the true gene order in at least $70 \%$ of the cases and has proven useful for marker development [7]. The high accuracy of this approach was confirmed by the data evaluated in the recent publication of a near-complete barley genome [6]. With barley being a very close relative and a model system for wheat, we expect similar accuracy for wheat chromosome $1 \mathrm{AS}$ and that the majority of the BAC contigs were placed in the correct linear order along the chromosome. However, using syntenybased anchoring does not allow to determine if a particular gene is placed correctly in the map. Thus, the physical map describes the probable order of genes, but the precise chromosomal location of any given gene of interest will have to be confirmed independently by genetic and/or physical mapping (e.g. radiation hybrid mapping).

\section{Level 3 Anchoring: Adding Genetic Markers to the Physical Map}

A total of 16 genetic markers for chromosome 1AS deposited at GrainGenes could be linked to IAS BAC-end or Illumina sequences (Table 1, see methods). These markers originate from eight different mapping projects and/or populations, two of them are composite/consensus maps (Table 1). This makes it difficult to compare and integrate specific positions on genetic maps because the same markers used in different populations can give different map positions. Eleven of the markers had been compiled in a consensus SSR map [53] and/or a composite map (WheatComposite2004-1A, Appels et al., unpublished, Table 1). To obtain a consensus genetic map, we calculated for each marker the average of available cM positions from different maps, being aware of the known limitations and problems of such an approach [54]. Markers and their genetic map positions relative to the telomere were then integrated with the synteny-based physical map (Figure 4). Two markers (whs179 and F640) were integrated based on local genetic maps of the Pm3 [29] and Lr10 loci [55], respectively.

The 16 genetic markers anchor 16 BAC contigs to a compiled genetic map of $50 \mathrm{cM}$ (Figure 4). The order of the genetic maps largely corresponds to the order of the BAC contigs that was inferred from synteny to Brachypodium, rice and sorghum. In only two cases (barc148 and fba393), the position on the genetic map contradicted the synteny-based anchoring. Synteny-based anchoring placed barc393 close to the centromere while it genetically maps close to the telomere. The inverse situation was found for marker barc148. Based on synteny, barc148 was anchored distantly at reference zipper gene 48 (Bradi4g38230). Further investigation showed that the gene was found in this position only in rice and Sorghum, while it is absent in Brachypodium. The homolog 
(Bradi4g38230) was inserted ("zipped in", see methods) based on the rice and sorghum data. We conclude that in this case, movement of this gene in the Brachypodium/wheat lineage led to incorrect anchoring of a BAC contig.

\section{Level 3 Anchoring: Distal Regions Show Higher Recombination Frequencies than Proximal Ones}

Interestingly, the distal regions of the transcriptome-based physical map showed a high density of genetic markers, especially in well-studied regions containing the Pm $3, \operatorname{Lrk} 10$ and $\operatorname{Lr} 10$ disease resistance loci. Indeed, there are 10 genetic markers clustered in the distal $10 \%$ of the physical map. However, on the genetic map, these 10 markers are spread out over more than $20 \mathrm{cM}$ (Figure 4). Three genetic markers psp3027, $\operatorname{barc} 148$ and $w m c 286$ all genetically mapped to the centromere region (Figure 4). In general, the centromere was shown to be largely recombination-free [56]. In the cases described here, the genetic centromere is spread out over almost $10 \mathrm{cM}$. This is because the centromere is at different $\mathrm{cM}$ position depending on the population that was used. Similarly, the $12 \mathrm{cM}$ genetic distance between the Pm3 and Lr10 loci in the distal region of the physical map should not be taken at face value because different populations were used to map these two genes $[29,30]$.

Although limited, the genetic data that we could integrate in the lAS map is nevertheless insightful and it has implications for future genetic mapping and breeding projects. Most importantly, it shows that large parts of the 1AS chromosome arm show very little recombination (at least in the genetic data that was available for this study). Previous studies on wheat chromosomes indicated that recombination frequencies are highest in distal regions while centromeric region show almost no recombination [57]. For example, on chromosome 3B, $42 \%$ of the physical map corresponds to only $2.2 \%$ of the genetic map in the centromeric regions [18]. On 1AS, approximately half of the $1 \mathrm{AS}$ genetic map represents only about 100-120 genes of the $1 \mathrm{~S}$ reference zipper. This means that half of all recombination events take place in a physical region that contains less than the distal $15 \%$ of genes. Thus, different approaches such as radiation hybrid mapping that are independent of recombination [58] will be necessary to precisely map most of the wheat chromosomes.

\section{Evaluation of the Chromosome 1AS Pm3 locus using Physical Contigs and Sequenced BACs}

To investigate the validity of the physical map at a smaller scale, we used the previously published sequence covering the Pm3 locus (accession number AY146587 [59]). This sequence has a size of approximately $178 \mathrm{~kb}$ and was isolated from wheat cultivar Chinese Spring and should therefore have its exact match in the lAS physical map. BAC end sequences and positive NimbleGen hybridisation probe sequences were used to align the published Pm3 BAC sequence to four 1AS BAC clones (TaaCsplAShA0068N15, TaaCsp1AShA0060M21, TaaCsp1AShA0041L1 1 and TaaCsp1AShA0068L13). These were placed in two different physical contigs (ltc132 and ltc5245). Interestingly, those two BAC contigs were placed directly adjacent to each other in the syntenybased anchoring (see above) and the published Pm3 BAC sequence links these two contigs together (Figure 6).

A total of 12 UniGene hybridisation probes were found on the two physical contigs. These could be assigned to two gene loci. Three of the probes correspond to the Pm3 gene while a second cluster of 7 probes corresponds to one or more glutenin genes (Figure 6). This is in agreement with previous studies, which reported the presence of multiple low-molecular weight glutenins at the Pm3 locus $[60,61]$.

To further characterise the glutenin gene cluster, we used the 7 EST UniGene probes that mapped to the glutenin locus in Blastn searches against the IWGSC survey sequences from flow sorted chromosomes. Five of the probes had their best matches in the 1AS Illumina dataset while two originate from chromosome 1BS. Phylogenetic analysis of all probe sequences plus previously published glutenin sequences suggested that a cluster of five genes is present on BAC contig ltc132. Furthermore, using the physical mapping data available from $\operatorname{ctg} 132$, we estimate the glutenin gene cluster to be between 52 and $84 \mathrm{~kb}$ away from the left end of the contig and $100 \mathrm{~kb}$ away from the Pm3 gene. Despite the rapid divergence of these loci, the estimation of $100 \mathrm{~kb}$ between the two gene loci compares well with the previous estimates in three different wheat ploidy levels [61].

The order of BAC-ends and NimbleGen hybridisation probe homologs on the published Pm3 sequence corresponds nearly perfectly with the order of BACs inferred by LTC. Furthermore the close physical association of the Pm3 gene with a glutenin cluster agrees with previously published genetic and physical mapping data $[62,63]$. These findings are strong indicators for a high quality and robustness of the LTC fingerprint assembly and the lAS physical map in general.

\section{Construction of a 3.8 Mbp Integrated Sequence Model}

Having Illumina sequences that completely cover the 1AS chromosome arm available through IWGSC, we aimed at integrating as much of the sequence data in the 1AS physical map. As described in the methods, we used BAC-end as well as NimbleGen UniGene sequences from anchored BAC contigs to identify the matching Illumina contigs. Here it must be noted that we did not mask repetitive sequences but applied very stringent criteria for the Blastn searches. This was done to allow anchoring of possible gene sequences that are located next to repetitive sequences on an Illumina contig (example in Figure 5a, case D).

In total, we anchored 852 Illumina sequence contigs to the 185 BAC contigs that were ordered in the level 2 and level 3 anchoring steps. The 852 Illumina contigs have a cumulative size of $3.82 \mathrm{Mbp}$ and therefore represent approximately $1.4 \%$ of the 1AS chromosome arm. The sequences were ordered in a single fasta flatfile according to their position in the physical map (i.e. the first sequence in the flatfile represents the one closest to the telomere). The definition line ("DE line") of each of the 852 Illumina sequences contains basic information on the main characteristics of the sequence (Example in Table 7). The sequence model contains a total of 444 gene sequences, which are annotated with their approximate positions.

\section{Conclusion}

In this study we aimed at integrating multiple different resources to produce a framework that can be used for future research and mapping projects, with the ultimate goal of a complete genomic sequence of the $1 \mathrm{AS}$ chromosome arm of wheat. The 1AS physical map as it stands now can serve research in multiple ways. For example, it provides practical "entry points" for genetic mapping projects. The anchored BAC-end and Illumina sequences can serve for the development of new genetic markers. Here, resources from 454 whole-genome sequencing [62] can be used to produce markers based on single nucleotide polymorphisms (SNPs). Once a target gene is genetically located, BAC contigs can provide direct access to candidate gene sequences. Furthermore, we were able to assign almost $11 \mathrm{Mbp}$ of BAC contigs to the centromeric region of 


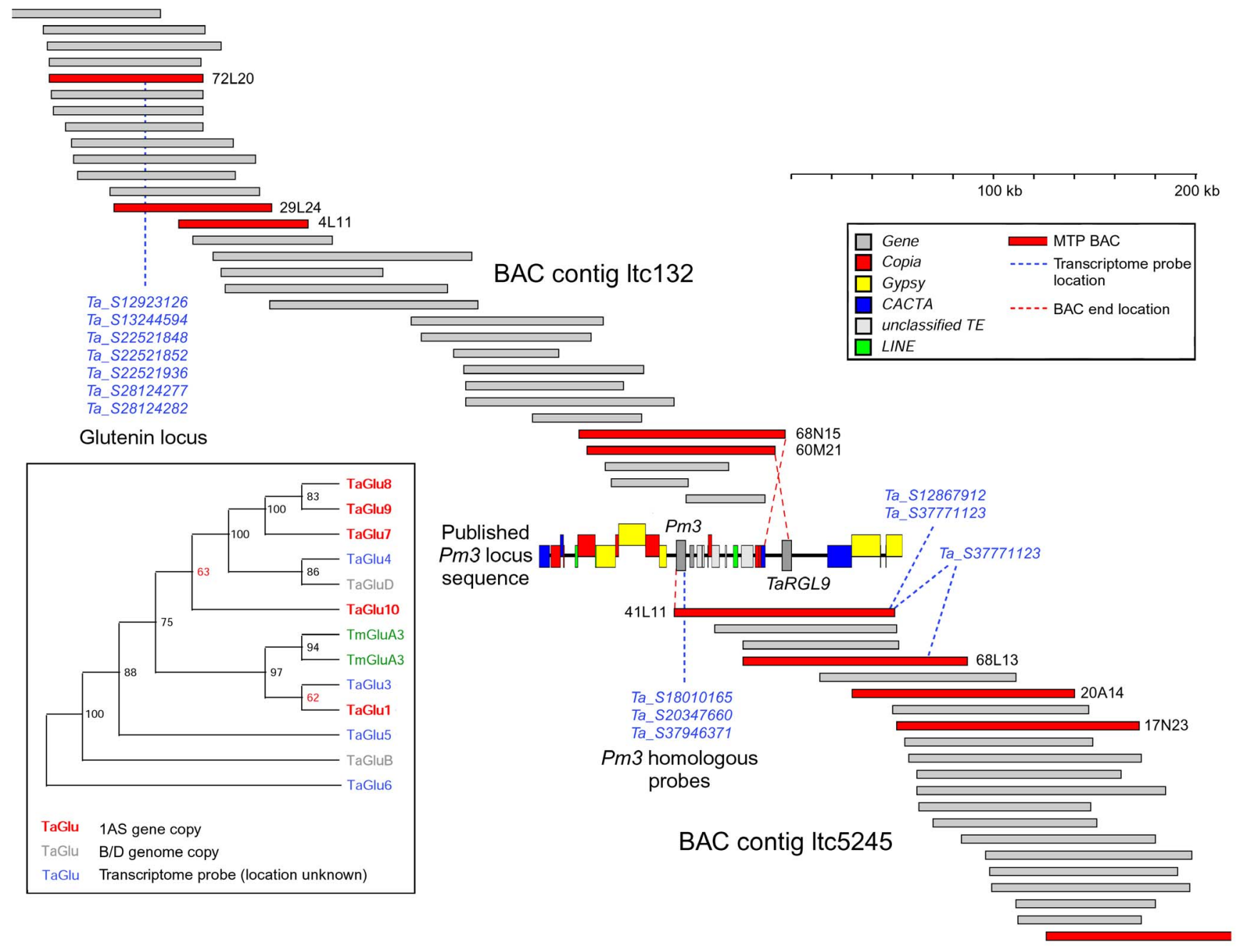

Figure 6. Small scale validation of the chromosome 1AS physical map using information from the previously published Pm3 powdery mildew resistance locus. Two assembled physical contigs (Itc132 and Itc5245) were linked together using a previously published $178 \mathrm{~kb}$ sequence from chromosome 1AS (cv. Chinese Spring) covering the the Pm3 locus (Wicker et al. 2007). Approximate locations of NimbleGen transcriptome hybridisation probes are shown in blue. The Pm3 and the low molecular weight (LMW) glutenin loci are known to be closely linked (Wicker et al. 2003; Wang et al. 2010). The inset shows a phylogenetic analysis that compares glutenin UniGene sequences with previously published glutenin genes from 1AS (TmGluA3, green), 1BS (TaGluB) and 1DS (TaGluD). doi:10.1371/journal.pone.0080272.g006

lAS. This is noteworthy as many important genes are located in the centromeres of wheat chromosome such as Ph1 [63]. The map also allows the reverse approach of identifying homologous regions of important chromosome 1AS genes in model systems, providing more efficient strategies in studying gene function [64,65].
To further advance the 1AS physical mapping project, it will be of great importance to integrate a much larger number of genetic markers. This will allow to move away from ordering of sequences based on synteny to model genomes to inferring sequence order based on actual wheat data. Next generation sequencing of sorted

Table 7. Information contained in the definition lines of Illumina sequences that were used for the integrated 1 AS sequence model in the order it is given in the definition line.

\begin{tabular}{lll}
\hline Name & Example & Explanation \\
\hline Identifier & 1AS_z_110 & Chromosome arm and zipper position \\
Illumina name & 1AS_c-5660802 & Original name of Illumina contig \\
Gene content & Bradi3g05850 282-1376 & Approximate position of gene(s) \\
Contig position & on ctg: $108-216$ & Position of respective BAC in contig [kb] \\
NimbleGen probe & signal =Ta_S12867912 & Name of probe that gave a signal \\
\hline doi:10.1371/journal.pone.0080272.t007 & &
\end{tabular}


chromosome arms from different wheat genotypes can be used as a source of genetic markers to accomplish this task in the near future [66]. Additionally with the decreasing costs of sequencing, a complete sequencing of the 1AS minimum tiling path in now conceivable and will be an essential step toward the ultimate goal of a complete genomic sequence of the 1AS chromosome arm of wheat.

\section{Supporting Information}

Figure S1 Number and distribution of UniGene probes that hybridised to BAC contigs. The $\mathrm{x}$-axis indicates the number of UniGene probes per BAC contig. The y-axis indicates how many $\mathrm{BAC}$ contigs contain the respective number of probes (e.g. $75 \mathrm{BAC}$ contigs contain produced hybridisation signals to exactly one UniGene probe). In total, 272 BAC contigs contain UniGene probes.

(PDF)

\section{References}

1. Gill BS, Appels R, Botha-Oberholster AM, Buell CR, Bennetzen JL, et al. (2004) A workshop report on wheat genome sequencing: International genome research on wheat consortium. Genetics 168: 1087-1096.

2. International Rice Genome Sequencing Project (2005) The map-based sequence of the rice genome. Nature 436: 793-800.

3. Paterson AH, Bowers JE, Bruggmann R, Dubchak I, Grimwood J, et al. (2009) The Sorghum bicolor genome and the diversification of grasses. Nature 457: 551556.

4. International Brachypodium Initiative (2010) Genome sequencing and analysis of the model grass Brachypodium distachyon. Nature 463: 763-768.

5. International Barley Genome Sequencing Consortium (2012) A physical, genetic and functional sequence assembly of the barley genome. Nature 491: 711-716.

6. Mayer KFX, Taudien S, Martis M, Simkova H, Suchankova P, et al. (2009) Gene content and virtual gene order of barley chromosome 1H. Plant Physiol 151: 496-505.

7. Mayer KFX, Martis M, Hedley PE, Simkovaa H, Liu H, et al. (2011) Unlocking the barley genome by chromosomal and comparative genomics. Plant Cell 23: 1249-1263.

8. Wicker T, Mayer KFX, Gundlach H, Martis M, Steuernagel B, et al. (2011) Frequent gene movement and pseudogene evolution is common to the large and complex genomes of wheat, barley, and their relatives. Plant Cell 23: 17061718 .

9. Choulet F, Wicker T, Rustenholz C, Paux E, Salse J, et al. (2010) Megabase level sequencing reveals contrasted organization and evolution patterns of the wheat gene and transposable element spaces. Plant Cell. 22: 1686-1701.

10. Bennett MD, Smith JB (1976) Nuclear DNA amounts in angiosperms. Philos Trans R Soc Lond B Biol Sci 274: 227-274.

11. Paux E, Roger D, Badaeva E, Gay G, Bernard M, et al. (2006) Characterizing the composition and evolution of homoeologous genomes in hexaploid wheat through BAC-end sequencing on chromosome 3B. Plant J 48: 463-474.

12. Arabidopsis Genome Initiative (2000) Analysis of the genome sequence of the flowering plant Arabidopsis thaliana. Nature 408: 796-815

13. Wei F, Coe E, Nelson W, Bharti AK, Engler F, et al. (2007) Physical and genetic structure of the maize genome reflects its complex evolutionary history. PLoS Genet 3: e123.

14. Wei F, Stein JC, Liang C, Zhang J, Fulton RS, et al. (2009) Detailed analysis of a contiguous 22-Mb region of the maize genome. PLoS Genet 5: e1000728.

15. Šafář J, Šimková H, Kubaláková M, Číhalíková J, Suchánková P, et al. (2010) Development of chromosome-specific BAC resources for genomics of bread wheat. Cytogenet Genome Res 129: 211-223.

16. Kubaláková M, Kovařová $\mathrm{P}$, Suchánková $\mathrm{P}$, Č́íhalíková J, Bartoš J, et al. (2005) Chromosome sorting in tetraploid wheat and its potential for genome analysis. Genetics 170: 823-829.

17. Doležel J, Kubaláková M, Paux E, Bartoš J, Feuillet C (2007) Chromosomebased genomics in the cereals. Chromosome Res 15: 51-66.

18. Paux E, Sourdille P, Salse J, Saintenac C, Choulet F, et al. (2008) A physical map of the 1-gigabase bread wheat chromosome 3B. Science 322: 101-104

19. Brenchley R, Spannagl M, Pfeifer M, Barker GL, D’Amore R, et al. (2012) Analysis of the bread wheat genome using whole-genome shotgun sequencing. Nature. 491: 705-710.

20. Soderlund C, Longden I, Mott R (1997) FPC: a system for building contigs from restriction fingerprinted clones. Comput Appl Biosci 13: 523-535.

21. Frenkel Z, Paux E, Mester D, Feuillet C, Korol A (2010) LTC: a novel algorithm to improve the efficiency of contig assembly for physical mapping in complex genomes. BMC Bioinformatics 11: 584.

\section{Acknowledgments}

We are grateful to Prof. B. S. Gill (Kansas State University, Manhattan, USA) for seeds of the double ditelosomic line $1 \mathrm{~A}$ of wheat T. aestivum $\mathrm{L}$. cv. Chinese Spring. We thank Jan Buchmann, Christopher Middleton, Simone Oberhaensli and Stefan Roffler for their competent support in computer programming and system administration. Assistance of Zdeňka Dubská, Jarmila Č́halíková, Marie Kubaláková, Romana Šperková and Jana Dostálová with chromosome sorting and BAC library construction is greatly appreciated.

\section{Author Contributions}

Conceived and designed the experiments: BK TW. Performed the experiments: HS JS JD FM SS FC CF EP HB IB RP SV TF WS. Analyzed the data: JB TW MS AK ZF. Contributed reagents/materials/ analysis tools: HS JS JD. Wrote the paper: BK JB TW.

22. Rustenholz C, Choulet F, Laugier C, Šafaář J, Šimková H, et al. (2011) A 3,000loci transcription map of chromosome $3 \mathrm{~B}$ unravels the structural and functional features of gene islands in hexaploid wheat. Plant Physiol 157: 1596-1608.

23. Lazo GR, Chao S, Hummel DD, Edwards H, Crossman CC, et al. (2004) Development of an expressed sequence tag (EST) resource for wheat (Triticum aestivum 1.): EST generation, UniGene analysis, probe selection and bioinformatics for a 16,000-locus bin-delineated map. Genetics 168: 585-593.

24. Qi LL, Echalier B, Chao S, Lazo GR, Butler GE, et al. (2004) A chromosome bin map of 16,000 expressed sequence tag loci and distribution of genes among the three genomes of polyploid wheat. Genetics 168: 701-712.

25. Rustenholz G, Hedley PE, Morris J, Choulet F, Feuillet C, et al. (2010) Specific patterns of gene space organisation revealed in wheat by using the combination of barley and wheat genomic resources. BMC Genomics 11: 714.

26. Gill KS, Gill BS, Endo TR, Boyko EV (1996) Identification and high-density mapping of gene-rich regions in chromosome group 5 of wheat. Genetics 143 : 1001-1012.

27. Breen J, Wicker T, Kong X, Zhang J, Ma W, et al. (2010) A highly conserved gene island of three genes on chromosome $3 \mathrm{~B}$ of hexaploid wheat: diverse gene function and genomic structure maintained in a tightly linked block. BMC Plant Biol 10: 98.

28. Gill KS, Gill BS (1991) A DNA fragment mapped within the submicroscopic deletion of $P h 1$, a chromosome pairing regulator gene in polyploid wheat. Genetics 129: 257-259.

29. Yahiaoui N, Srichumpa P, Dudler R, Keller B (2004) Genome analysis at different ploidy levels allows cloning of the powdery mildew resistance gene Pm $3 b$ from hexaploid wheat. Plant J 37: 528-538.

30. Feuillet C, Travella S, Stein N, Albar L, Nublat A, et al. (2003) Map-based isolation of the leaf rust disease resistance gene $\mathrm{Lr} 10$ from the hexaploid wheat (Triticum aestivum L.) genome. Proc Natl Acad Sci U S A 100: 15253-15258.

31. Ozdemir N, Cloutier S (2005) Expression analysis and physical mapping of lowmolecular-weight glutenin loci in hexaploid wheat (Triticum aestivum L.). Genome 48: 401-410.

32. Spielmeyer W, Richards RA (2004) Comparative mapping of wheat chromosome $1 \mathrm{AS}$ which contains the tiller inhibition gene (tin) with rice chromosome 5S. Theor Appl Genet 109: 1303-1310.

33. McFadden HG, Lehmensiek A, Lagudah ES (2006) Resistance gene analogues of wheat: molecular genetic analysis of ESTs. Theor Appl Genet 113: 987-1002.

34. Vrána J, Kubaláková M, Šimková H, Číhalíková J, Lysák MA, et al. (2000) Flow-sorting of mitotic chromosomes in common wheat (Triticum aestivum L.). Genetics 156: 2033-2041.

35. Šimková H, Č́íhalíková J, Vrána J, Lysák MA, Doležel J (2003) Preparation of HMW DNA from plant nuclei and chromosomes isolated from root tips. Biol. Plant. 46: 369-373.

36. Janda J, Šafář J, Kubaláková M, Bartoš J, Kovářová P, et al. (2006) Advanced resources for plant genomics: BAC library specific for the short arm of wheat chromosome 1B. Plant J. 47: 977-986.

37. Šimková H, Šafář J, Kubaláková M, Suchánková P, Číhalíková J, et al. (2011) BAC libraries from wheat chromosome 7D: efficient tool for positional cloning of aphid resistance genes. J. Biomed. Biotechnol. 2011: 302543.

38. Ding Y, Johnson MD, Chen WQ, Wong D, Chen YJ, et al. (2001) Five-colorbased high-information-content fingerprinting of bacterial artificial chromosome clones using type IIS restriction endonucleases. Genomics 74: 142-154.

39. Scalabrin S, Morgante M, Policriti A (2009) Automated fingerprint background removal: FPB. BMC Bioinformatics 10: 127.

40. You FM, Luo MC, Gu YQ, Lazo GR, Deal K, et al. (2007) GenoProfiler: batch processing of high-throughput capillary fingerprinting data. Bioinformatics 23 : $240-242$. 
41. Nelson W, Soderlund C (2009) Integrating sequence with FPC fingerprint maps. Nucleic Acids Res 37: e36.

42. Paux E, Legeai F, Guilhot N, Adam-Blondon AF, Alaux M, et al. (2008) Physical mapping in large genomes: accelerating anchoring of BAC contigs to genetic maps through in silico analysis. Funct Integr Genomics 8: 29-32.

43. Peng JH, Zadeh H, Lazo GR, Gustafson JP, Chao S, et al. (2004) Chromosome bin map of expressed sequence tags in homoeologous group 1 of hexaploid wheat and homoeology with rice and Arabidopsis. Genetics 168: 609-623.

44. Nelson WM, Bharti AK, Butler E, Wei F, Fuks G, et al. (2005) Whole-genome validation of high-information-content fingerprinting. Plant Physiol 139: 27-38.

45. Vitulo N, Albiero A, Forcato C, Campagna D, Pero FD, et al. (2011) First survey of the wheat chromosome $5 \mathrm{~A}$ composition through a next generation sequencing approach. PLoS One 6: e26421.

46. Presting GG, Malysheva L, Fuchs J, Schubert I (1998) A Ty3/gypsy retrotransposon-like sequence localizes to the centromeric regions of cereal chromosomes. Plant J 16: 721-728.

47. Houben A, Schroeder-Reiter E, Nagaki K, Nasuda S, Wanner G, et al. (2007) CENH3 interacts with the centromeric retrotransposon Cereba and GC-rich satellites and locates to centromeric substructures in barley. Chromosoma 116: 275-283.

48. Vicient CM, Kalendar R, Anamthawat-Jansson K, Schulman AH (1999) Structure, functionality, and evolution of the BARE-1 retrotransposon of barley. Genetica 107: 53-63.

49. Kalendar R, Tanskanen J, Immonen S, Nevo E, Schulman AH (2000) Genome evolution of wild barley (Hordeum spontaneum) by BARE-1 retrotransposon dynamics in response to sharp microclimatic divergence. Proc Natl Acad Sci U S A 97: 6603-6607.

50. Soleimani VD, Baum BR, Johnson DA (2006) Quantification of the retrotransposon $B A R E-1$ reveals the dynamic nature of the barley genome. Genome 49: 389-396.

51. Wicker T, Taudien S, Houben A, Keller B, Graner A, et al. (2009) A wholegenome snapshot of 454 sequences exposes the composition of the barley genome and provides evidence for parallel evolution of genome size in wheat and barley. Plant J 59: 712-722.

52. Middleton CP, Stein N, Keller B, Kilian B, Wicker T (2012) Comparative analysis of genome composition in Triticeae reveals strong variation in transposable element dynamics and nucleotide diversity. Plant J.

53. Somers DJ, Isaac P, Edwards K (2004) A high-density microsatellite consensus map for bread wheat (Triticum aestivum L.). Theor Appl Genet 109: 1105-1114.

54. Ronin Y, Mester D, Minkov D, Belotserkovski R, Jackson BN, et al. (2012) Twophase analysis in consensus genetic mapping. G3 (Bethesda). 2: 537-549.

55. Wicker T, Stein N, Albar L, Feuillet C, Schlagenhauf E, et al. (2001) Analysis of a contiguous $211 \mathrm{~kb}$ sequence in diploid wheat (Triticum monococcum L.) reveals multiple mechanisms of genome evolution. Plant J 26: 307-316.
56. Yan H, Jin W, Nagaki K, Tian S, Ouyang S, et al. (2005) Transcription and histone modifications in the recombination-free region spanning a rice centromere. Plant Cell 17: 3227-3238.

57. Akhunov ED, Goodyear AW, Geng S, Qi LL, Echalier B, et al. (2003) The organization and rate of evolution of wheat genomes are correlated with recombination rates along chromosome arms. Genome Res 13: 753-763.

58. Kalavacharla V, Hossain K, Gu Y, Riera-Lizarazu O, Vales MI, et al. (2006) High-resolution radiation hybrid map of wheat chromosome 1D. Genetics 173: 1089-1099.

59. Wicker T, Yahiaoui N, Keller B (2007) Contrasting rates of evolution in Pm3 loci from three wheat species and rice Genetics 177: 1207-1216.

60. Wang ZN, Huang XQ Cloutier S (2010) Recruitment of closely linked genes for divergent functions: the seed storage protein (GLU-3) and powdery mildew (Pm3) genes in wheat (Triticum aestivum L.). Funct Integr Genomics 10: 241-251.

61. Wicker T, Yahiaoui N, Guyot R, Schlagenhauf E, Liu ZD, et al. (2003) Rapid genome divergence at orthologous low molecular weight glutenin loci of the A and $\mathrm{A}^{\mathrm{m}}$ genomes of wheat. Plant Cell 15: 1186-1197.

62. Allen AM, Barker GLA, Berry ST, Coghill JA, Gwilliam R, et al. (2011) Transcript-specific, single-nucleotide polymorphism discovery and linkage analysis in hexaploid bread wheat (Triticum aestivum L.). Plant Biotechnol J 9: 1086-1099.

63. Griffiths S, Sharp R, Foote TN, Bertin I, Wanous M, et al. (2006) Molecular characterization of $P h 1$ as a major chromosome pairing locus in polyploid wheat. Nature 439: 749-752.

64. Febrer M, Goicoechea JL, Wright J, McKenzie N, Song X, et al. (2010) An integrated physical, genetic and cytogenetic map of Brachypodium distachyon, a model system for grass research. PLoS One 5: e13461.

65. Brkljacic J, Grotewold E, Scholl R, Mockler T, Garvin DF, et al. (2011) Brachypodium as a model for the grasses: today and the future. Plant Physiol 157: $3-13$.

66. Shatalina M, Wicker T, Buchmann JP, Oberhaensli S, Simkovaa H, et al. (2013) Genotype-specific SNP map based on whole chromosome 3B sequence information from wheat cultivars Arina and Forno. Plant Biotechnol J 11: 2332.

67. Dubcovsky J, Luo M, Dvorak J (1995) Differentiation between homoeologous chromosomes $1 \mathrm{~A}$ of wheat 7 and $1 \mathrm{~A}^{\mathrm{m}}$ of Triticum monococcum and its recognition by the wheat Ph1 locus. Proc Natl Acad Sci U S A 92: 6645-6649.

68. Paillard S, Schnurbusch T, Winzeler M, Messmer M, Sourdille P, et al. (2003) An integrative genetic linkage map of winter wheat (Triticum aestivum L.). Theor Appl Genet 107: 1235-1242.

69. Xue S, Zhang Z, Lin F, Kong Z, Cao Y, et al. (2008) A high-density intervarietal map of the wheat genome enriched with markers derived from expressed sequence tags. Theor Appl Genet 117: 181-189.

70. Nelson JC, Sorrells ME, Deynze AEV, Lu YH, Atkinson M, et al. (1995) Molecular mapping of wheat : major genes and rearrangements in homoeologous groups 4, 5, and 7. Genetics 141: 721-731. 